\title{
Mathematical modeling of contact tracing as a control strategy of Ebola Virus Disease
}

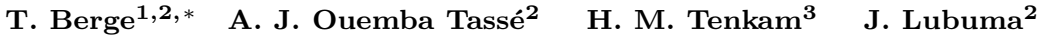 \\ ${ }^{1}$ Department of Mathematics and Applied Mathematics, University of Pretoria \\ Private Bag X20, Pretoria 0028, South Africa \\ ${ }^{2}$ Department of Mathematics and Computer Science, University of Dschang, \\ P. O. Box 67, Dschang, Cameroon \\ berge.tsanou@up.ac.za,bergetsanou@yahoo.fr ${ }^{\dagger}$ \\ ${ }^{3}$ Department of Mathematics and Applied Mathematics, North-West University, \\ Private Bag X1290 Potchefstroom 2520, South Africa
}

\begin{abstract}
More than 20 outbreaks of Ebola virus disease have occurred in Africa since 1976, and yet no adequate treatment is available. Hence, prevention, control measures and supportive treatment remain the only means to avoid the disease. Amongst these measures, contact tracing occupies a prominent place. In this paper, we propose a simple mathematical model that incorporates imperfect contact tracing, quarantine and hospitalization (or isolation). The control reproduction number $\mathcal{R}_{c}$ of each sub-model and for the full model are computed. Theoretically, we prove that when $\mathcal{R}_{c}$ is less than one, the corresponding model has a unique globally asymptotically stable disease-free equilibrium. Conversely, when $\mathcal{R}_{c}$ is greater than one, the disease-free equilibrium becomes unstable and a unique globally asymptotically stable endemic equilibrium arises. Furthermore, we numerically support the analytical results and assess the efficiency of different control strategies. Our main observation is that, to eradicate EVD, the combination of high contact tracing (up to 90\%) and effective isolation is better than all other control measures, namely: (1) perfect contact tracing, (2) effective isolation or full hospitalization, (3) combination of medium contact tracing and medium isolation.
\end{abstract}

Keywords: Ebola; Contact tracing; Quarantine; Isolation; Control Reproduction Number; Global Stability.

\section{Introduction}

The name Ebola comes from a river in the city of Yambuku at the North of the Democratic Republic of Congo (DRC) [2]. It is in the hospital of that city the first

*Corresponding author

†bergetsanou@yahoo.fr 
patient of Ebola virus disease was diagnosed in 1976. Since then, there have been more than 20 outbreaks with the deadliest recorded in Western Africa in 20132015 and the must recent in 2018 in DRC $[1,15,17,44,79]$. It is well known that, human-to-human transmission of EVD occurs when a susceptible human comes into contact with the blood secretions or other bodily fluids of an infected one $[1,3,9,20,34,42,47,55,73]$.

The resurgence of EVD and its case fatality rate of about $25 \%$ to $90 \%$ gave rise to many consequences and urged the United Nation Organization to qualify it as "threat to international peace and security" $[1,2,35,42,83]$. Moreover, an intensive scientific research activity was triggered and recommended the healthcare organizations to adopt an outfit of control measures to hold back and contain the 2013-2015 epidemic [10,30,36, 42, 88]. Amongst these measures, entry-screening in the countries of Guinea, Liberia, Sierra Leone and ring vaccination in Guinea were implemented, but their impact in containing the disease remained mitigated [53,59]. Therefore, intensive contact tracing took place as an additional control strategy as it is known to be the most efficient measure applied so far to curtain almost all EVD outbreaks $[23,25,30,65]$. Hence, the main purpose of this manuscript.

Actually, contact tracing is a mitigation strategy that aims at immediately detecting the next-generation cases during the spreading of an infectious disease $[12,24]$. In West Africa and in many other countries, the persons who were exposed to infected cases were traced and quarantined $[12,24]$. Moreover, tracing and quarantining hundreds of people who had contact with two confirmed cases helped to prevent the spread of EVD in Nigeria and Senegal [24]. This shows how efficient contact tracing could be. It therefore became the focus and a field of interest that stimulated an exciting scientific research activities, amongst which mathematical modeling could and should have a prominent role to play $[7,8,11,23,42,51,72,73,87]$.

As far as EVD is concerned, many mathematical models have been developed to explain human-to-human transmissions $[1,5,6,12,15,17,20,28,37,38,49,56,62$, $68,69,71,73,77,78,79,82,83,87]$. Some of them included both direct and indirect transmissions $[15,17,78,79]$, but very few investigated the control strategies such as vaccination, quarantine, isolation $[11,20,47,72,73,83,87]$. To the best of the authors knowledge, very few mathematical models dealt with contact tracing [23,42]. Moreover, although these latter two works have offered insights into the understanding, the control and eradication of EVD, they have exhibited some drawbacks: In [23], the authors did not considered the fact that, traced and tested positive individuals should be hospitalized, and once at the hospital they could continue to transmit the disease (see for instance $[61,67,85]$ ). Concerning [42], from our understanding: (1) There were some confusion between the compartment of suspected and that of probables. (2) The distinction between quarantined and isolated individuals wasn't clear enough. (3) It was not well understood how some probables cases were treated without being hospitalized. (4) Assuming that infected individuals who died naturally could not transmit EVD wasn't realistic. The purpose of our work is to fill some of the gaps mentioned above by developing a contact tracing model that sim- 
plifies the one in [42] in order to allow a better understanding of the transmission processes of EVD and give an insightful mathematical analysis.

The propounded model is analyzed in three different aspects: (i)- perfect contact tracing, (ii)- no contact tracing at all, (iii)- imperfect contact tracing. In all cases, we show that, either the disease dies out whenever the control reproduction number is less than one (i.e. the disease-free equilibrium (DFE) is globally asymptotically stable (GAS)), or it persists whenever the said control reproduction number is greater than one (i.e. there exits and additional equilibrium: the endemic equilibrium which is GAS) and the DFE is unstable in this latter case. Moreover, we conduct numerical simulations to illustrate theoretical results. Furthermore, focusing on the efficacy of contact tracing and isolation, we first show numerically that the number of infected individuals decreases over time as the contact tracing parameter increases, thus suggesting that, a good implementation of contact tracing follow by a quarantine of suspected individuals can contribute to a important reduction of the epidemic level of EVD. Secondly, we illustrate the favorable effect of reducing the contacts between susceptibles and hospitalized/isolated in fighting EVD. Finally, we assess the impacts of: (i)-high contact tracing (up to 90\%) and full hospitalization, (ii)perfect contact tracing alone, (iii)-medium of both contact tracing and isolation, and (iv)-full hospitalization without contact tracing, on EVD dynamics. Our investigations suggest that, among the above four control measures, high contact tracing (up to 90\%) and full hospitalization seems to be the best to fight against EVD in the sense that its implementation leads to less infected compared with the three other strategies.

The rest of this paper is organized as follows. In Section 2, the full and simplified models are derived. The qualitative analysis of the models with perfect contact tracing, without contact tracing and with imperfect contact tracing are completely addressed in Section 3. Section 4 deals with the sensitivity analysis of the control reproduction number. Numerical simulations are provided in Section 5 to support the analytical results as well as to assess the impacts of contact tracing and isolation. The discussion, concluding remarks and perspectives are provided in Section 6 .

\section{Model development}

\subsection{Main assumptions}

Many of the following assumptions are given as a realistic attempt to fill the gaps mentioned above in $[23,42]$.

(1) For simplicity, we assume the mass action incidence.

(2) Not all exposed individuals to infectives are necessary traced and identified.

(3) The infected individuals can only recover when they are hospitalized, and those quarantined individuals tested positive are immediately hospitalized as well.

(4) We assume that the identification of a contact is instantaneous (i.e. people are immediately traced after being exposed to an infective). However, it would be 
more realistic to assume that there is a time lag between a contact and its identification. This last assumption would lead to a delay differential system which, for the sake of simplicity is postponed for further work.

(5) For our model to be meaningful, it is necessarly to assume that, initially $(t=0)$, they are already few infectives (otherwise the contact tracing which is the main focus of our work will not be implementable).

In order to derive the model equations and after given the main assumptions, we shall carefully select and motivate the choices of the model variables.

\subsection{Model variables}

At time $t$, the human population is subdivided into mutually exclusive classes as follows:

- $S(t)$ : Individuals not yet infected and able to acquire EVD.

- $Q(t)$ : Individuals who were exposed to infectives, identified and quarantined before showing EVD symptoms [47]. Note that, quarantine is used to separate and restrict the movements of healthy persons who may have been exposed to a communicable disease in order to see whether they will exhibit the specific disease symptoms. These people may have been exposed to the infection and do not know it, or they may have acquired the disease pathogen but do not show symptoms $[31,48]$. Therefore, those individuals in the $Q$-class who do not show symptoms after 21 days (the maximun latent period for EVD $[3,22,55]$ ) will be considered susceptibles [30,63], whereas those who are tested positive will be hospitalized.

- $U(t)$ : Unidentified individuals who have been in contact with infectives. They can not be suspected as long as they do not show disease symptoms. This class of individuals encompasses those are infected, those in the latent stage, as well as healthcare workers.

- $I(t)$ : Infected individuals who exhibit EVD symptoms but are not yet in the hospital.

- $H(t)$ : Infected individuals in the treatment centers (i.e the hospitalized/isolated individuals). Let us recall that, isolation is a process by which infected individuals are separated from those who are healthy in order to undergo proper treatment $[31,48]$. We assume that, the Ebola-deceased individuals who died at the hospital are safely buried and therefore do not transmit the infection.

- $R(t)$ : Recovered and removed individuals. Even though up to now, there is no homologated treatment for EVD, some patients have recovered after receiving supportive treatments $[3,47]$. Moreover, since it is documented that recovered individuals develop antibodies that last for at least 10 years [27,47], we assume that they are permanently immunized.

To achieve the model formulation and provide mathematical equations, one needs to identify and define the specific model parameters. 


\subsection{Model parameters and derivation of model equations}

We denote by $\pi$, the number of susceptible individuals recruited per unit time (day) as a result of births or immigration and $\mu$ the common natural mortality rate of all individuals. The disease is transmitted through direct contact between susceptible individuals and: (a) infected individuals $(I)$, (b) hospitalized individuals $(H),(\mathrm{c})$ Ebola-deceased individuals (those died out of the hospital) and not safely buried. Let $\beta$ be the contact rate between susceptibles $(S)$ and infected $(I)$, and $\varepsilon_{h}$ the modification parameter for that contact rate between susceptibles and hospitalized individuals $(H)$. Since hospitalized individuals are isolated, it is reasonable to assume that $0 \leq \varepsilon_{h} \leq 1$. After contact between susceptibles and infectives, a proportion $p$ of these probables cases are identified, traced and quarantined [25]. Based on the medical tests, these quarantined individuals are either hospitalized (positive test) or considered susceptibles after 21 days (negative test). Let $\lambda_{q}$ be the exit rate from the quarantine class $Q$ to both classes $S$ and $H$. A proportion $p_{q s} \lambda_{q}$ of the quarantined goes back to the $S$-compartment, whereas the remaining proportion $\left(1-p_{q s}\right) \lambda_{q}$ enters class $H$. Similarly, $(1-p)$ is the proportion of untraced/unidentified individuals who enter the $U$-compartment. Of these individuals, a rate $\lambda_{u}$ exits the $U$-compartment so that $p_{u s} \lambda_{u} U$ and $\left(1-p_{u s}\right) \lambda_{u} U$ are the numbers of unidentified individuals who enter classes $S$ and $I$, respectively. Individuals in class $I$ move to class $H$ at rate $\eta_{i}$ and die due to EVD at rate $d_{i}$. Note that $\eta_{i}$ can increase as the number traced people increases [30]. Since the infected individuals who die out of the hospital can still transmit the disease, we assume that, among the $\left(d_{i}+\mu\right) I$ dead individuals, a proportion $\theta$ is safely buried, while the remaining proportion $(1-\theta)$ can transmit the infection during funerals. Let $\beta^{\prime}$ be the effective contact rate between susceptibles and Ebola-deceased. We assume $\beta^{\prime}=\beta \nu$, where $\nu(0 \leq \nu \leq 1)$ is the modification parameter which accounts for the fact that the number of contacts with a dead individual is less than that with an alive person. Individuals of class $H$ die due to EVD at rate $d_{h}$ and recover at rate $\gamma$ per unit time. We assume that the model parameters are nonnegative.

The transmission diagram is schematized in Figure 1 and the corresponding model equations are given in system (2.1) below.

$$
\left\{\begin{array}{l}
S^{\prime}=\pi-\beta_{0} S(I+\varepsilon H)+p_{q s} \lambda_{q} Q+p_{u s} \lambda_{u} U-\mu S \\
Q^{\prime}(t)=p \beta_{0} S(I+\varepsilon H)-\lambda_{q} Q-\mu Q \\
U^{\prime}(t)=(1-p) \beta_{0} S(I+\varepsilon H)-\lambda_{u} U-\mu U \\
I^{\prime}(t)=\left(1-p_{u s}\right) \lambda_{u} U-\left(\eta_{i}+d_{i}+\mu\right) I \\
H^{\prime}(t)=\left(1-p_{q s}\right) \lambda_{q} Q+\eta_{i} I-\left(d_{h}+\mu+\gamma\right) H \\
R^{\prime}(t)=\gamma H-\mu R
\end{array}\right.
$$

where, for notational elegance, we have denoted:

$$
\beta_{0}=\beta\left(1+\nu(1-\theta)\left(d_{i}+\mu\right)\right) \text { and } \varepsilon=\frac{\beta \varepsilon_{h}}{\beta_{0}} .
$$

Table 1 summarizes the model parameters and their biological meanings. 


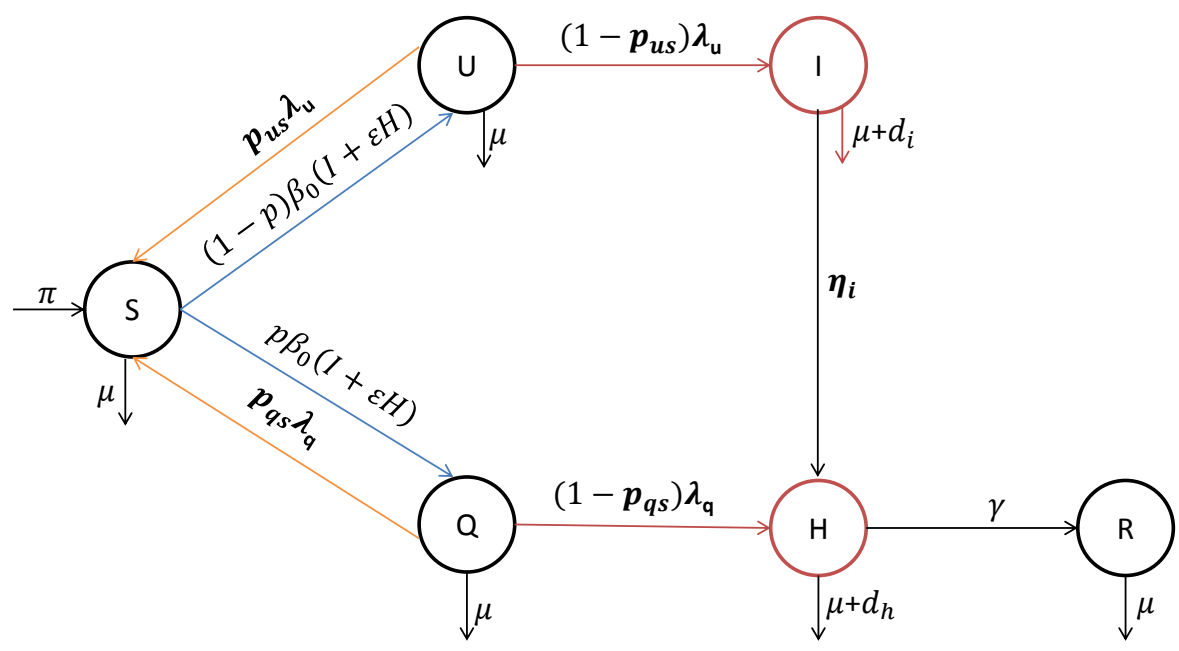

Fig. 1. Transmission diagram of the model

\begin{tabular}{|ll|}
\hline Parameter & Epidemiological interpretation \\
\hline$\mu$ & Natural mortality rate. \\
$p$ & Number of susceptible individuals recruited per unit time. \\
$\beta$ & Proportion of individuals who have been in contact with infectives and \\
$\beta^{\prime}$ & be quarantined. \\
$\nu$ & Contact rate of susceptibles with individuals of the $I$ compartment. \\
$\varepsilon_{h}$ & Modification parameter such that $\beta^{\prime}=\beta \nu$. \\
$\lambda_{u}$ & Modification factor representing the reduction of contacts between \\
$p_{u s} \lambda_{u}$ & Susceptibles and hospitalized individuals (i.e. efficacy of isolation). \\
$\lambda_{q}$ & Proportion of individuals of $U$ who leave this compartment to \\
$p_{q s} \lambda_{q}$ & Proportion of individuals of $U$ who leave this compartment to \\
$\eta_{i}$ & Proportion of individuals of $Q$ who leave this compartment to \\
$d_{i}$ & Poth compartment $S$ and $H$. \\
$d_{h}$ & Compartment $S$. \\
$\gamma$ & Hospitalization rate of infected individuals. \\
$\theta$ & Mortality rate due to EVD of individuals of class $I$. \\
& Mortality rate due to EVD of individuals of class $H$. \\
& Propory rate of hospitalized individuals. \\
\end{tabular}

Table 1. Model parameters and their epidemiological interpretations. 


\section{Remark 2.1.}

- If all the contacts are identified $(p=1)$ and all the infected individuals hospitalized, then the contact tracing is perfect. Thus, model (2.1) reduces to $\left(\beta=\beta_{0}, \varepsilon=\varepsilon_{h}\right)$ :

$$
\left\{\begin{array}{l}
S^{\prime}=\pi-\beta \varepsilon_{h} S H+p_{q s} \lambda_{q} Q-\mu S \\
Q^{\prime}(t)=\beta \varepsilon_{h} S H-\lambda_{q} Q-\mu Q \\
H^{\prime}(t)=\left(1-p_{q s}\right) \lambda_{q} Q-\left(d_{h}+\mu+\gamma\right) H \\
R^{\prime}(t)=\gamma H-\mu R
\end{array}\right.
$$

- Otherwise, if $p=0$ (no contact tracing at all), then class $U$ gathers all the individuals who had contact with infectious and model (2.1) becomes

$$
\left\{\begin{array}{l}
S^{\prime}=\pi-\beta_{0} S(I+\varepsilon H)+p_{u s} \lambda_{u} U-\mu S \\
U^{\prime}(t)=\beta_{0} S(I+\varepsilon H)-\lambda_{u} U-\mu U \\
I^{\prime}(t)=\left(1-p_{u s}\right) \lambda_{u} U-\left(\eta_{i}+d_{i}+\mu\right) I \\
H^{\prime}(t)=\eta_{i} I-\left(d_{h}+\mu+\gamma\right) H \\
R^{\prime}(t)=\gamma H-\mu R
\end{array}\right.
$$

The two specific sub-models mentioned in Remark 2.1 are theoretically analyzed in the following section, as well as the full model (2.1).

\section{Mathematical analysis}

\subsection{Mathematical analysis of model (2.2)}

The well-posedness of model (2.2) is postponed to Section 3.3 as a special case of system (2.1) when $p=1$. Thus, the compartments $U$ and $I$ are empty and must be removed from the model.

The following proposition is straightforward and establishes the existence of equilibria of model (2.2).

Proposition 3.1. The control reproduction number for model (2.2) is

$$
\mathcal{R}_{c}^{Q}=\frac{\beta \varepsilon_{h} \pi \lambda_{q}\left(1-p_{q s}\right)}{\mu\left(\lambda_{q}+\mu\right)\left(d_{h}+\mu+\gamma\right)} .
$$

Furthermore,

(i) If $\mathcal{R}_{c}^{Q} \leq 1$, there exists a unique disease-free equilibrium $E_{0}^{Q}$ given by

$$
E_{0}^{Q}=\left(\frac{\pi}{\mu}, 0,0,0\right) .
$$

(ii) If $\mathcal{R}_{c}^{Q}>1$, there are two equilibria: the DFE and a unique endemic equilibrium 
$E_{1}^{Q}$ whose components $\left(S_{q}^{*}, Q_{q}^{*}, H_{q}^{*}, R_{q}^{*}\right)$ are:

$$
\left\{\begin{aligned}
S_{q}^{*} & =\frac{\left(\lambda_{q}+\mu\right)\left(d_{h}+\mu+\gamma\right)}{\beta \varepsilon_{h} \lambda_{q}\left(1-p_{q s}\right)} \\
H_{q}^{*} & =\frac{\mu\left(\lambda_{q}+\mu\right)\left(\mathcal{R}_{c}^{Q}-1\right)}{\beta \varepsilon_{h}\left(\left(1-p_{q s}\right) \lambda_{q}+\mu\right)} \\
Q_{q}^{*} & =\frac{\left(d_{h}+\mu+\gamma\right) \mu\left(\lambda_{q}+\mu\right)\left(\mathcal{R}_{c}^{Q}-1\right)}{\left.\left(1-p_{q s}\right) \lambda_{q} \beta \varepsilon_{h}\left(\left(1-p_{q s}\right) \lambda_{q}+\mu\right)\right)} \\
R_{q}^{*} & =\frac{\gamma \mu\left(\lambda_{q}+\mu\right)\left(\mathcal{R}_{c}^{Q}-1\right)}{\mu \beta \varepsilon_{h}\left(\left(1-p_{q s}\right) \lambda_{q}+\mu\right)} .
\end{aligned}\right.
$$

We establish below the GAS of the DFE in order to show that the disease dies out completely whenever the control reproduction number $\mathcal{R}_{c}^{Q}$ is below one.

Theorem 3.2. The DFE is GAS if $\mathcal{R}_{c}^{Q} \leq 1$ and is unstable if $\mathcal{R}_{c}^{Q}>1$.

Proof: Consider the linear Lyapunov function candidate

$$
L_{q}=Q+\alpha H,
$$

where $\alpha>0$ is a positive number to be determined.

The derivative of $L_{q}$ in the direction of vector field gives:

$$
\dot{L}_{q}=Q\left[-\left(\lambda_{q}+\mu\right)+\left(1-p_{q s}\right) \lambda_{q} \alpha\right]+H\left[\frac{\beta \varepsilon_{h} \pi}{\mu}-\left(d_{h}+\mu+\gamma\right) \alpha\right] .
$$

We choose $\alpha$ such that $-\left(\lambda_{q}+\mu\right)+\left(1-p_{q s}\right) \lambda_{q} \alpha=0$. That is $\alpha=\frac{\lambda_{q}+\mu}{\left(1-p_{q s}\right) \lambda_{q}}$.

Thus,

$$
\begin{aligned}
\dot{L}_{q} & =H\left[\frac{\beta \varepsilon_{h} \pi}{\mu}-\frac{\left(d_{h}+\mu+\gamma\right)\left(\lambda_{q}+\mu\right)}{\left(1-p_{q s}\right) \lambda_{q}}\right] \\
& =\frac{\mu\left(\lambda_{q}+\mu\right)\left(d_{h}+\mu+\gamma\right)\left(\mathcal{R}_{c}^{Q}-1\right)}{\mu\left(1-p_{q s}\right) \lambda_{q}} H \\
& \leqslant 0 \quad \text { if } \quad \mathcal{R}_{c}^{Q} \leqslant 1 .
\end{aligned}
$$

Moreover, the largest invariant subset contained in the set $\left\{X \in \mathbb{R}_{+}^{4} / \dot{L}_{q}(X)=\right.$ $0\}$ when $\mathcal{R}_{c}^{Q} \leq 1$ is $\left\{E_{0}^{Q}\right\}$. Thus, the conclusion follows by LaSalle's Invariance Principle [54].

We shall prove the GAS of the endemic equilibrium for the special case where traced individuals are precisely those who had contact with infectives and will effectively develop the disease. That is, no quarantined individual becomes susceptible (i.e. $p_{q s}=0$ ). One should note that $\mathcal{R}_{c}^{Q}=\mathcal{R}_{c}^{Q}\left(p_{q s}\right)$.

Theorem 3.3. Assume $p_{q s}=0$, if $\mathcal{R}^{Q}=\mathcal{R}_{c}^{Q}(0)>1$, then the endemic equilibrium $E_{1}^{Q}$ of system (2.2) is GAS.

Proof: Consider the Volterra-Lyapunov candidate function :

$$
L_{q 1}=a_{1}\left(S-S^{*} \ln S\right)+b_{1}\left(Q-Q^{*} \ln Q\right)+c_{1}\left(H-H^{*} \ln H\right),
$$


where $a_{1}, b_{1}, c_{1}$ are positive numbers to be determined. The time derivative of $L_{q 1}$ along the trajectories of system (2.2) yields

$$
\begin{aligned}
\dot{L}_{q 1}= & a_{1}\left(1-\frac{S^{*}}{S}\right) S^{\prime}+b_{1}\left(1-\frac{Q^{*}}{Q}\right) Q^{\prime}+c_{1}\left(1-\frac{H^{*}}{H}\right) H^{\prime} \\
= & a_{1}\left(1-\frac{S^{*}}{S}\right)\left[\pi-\beta_{0} \varepsilon H S-\mu S\right]+b_{1}\left(1-\frac{Q^{*}}{Q}\right)\left[\beta_{0} \varepsilon H S-\left(\lambda_{q}+\mu\right) Q\right] \\
& +c_{1}\left(1-\frac{H^{*}}{H}\right)\left[\lambda_{q} Q-\left(d_{h}+\mu+\gamma\right) H\right] \\
= & a_{1}\left(1-\frac{S^{*}}{S}\right)\left[\beta_{0} \varepsilon H^{*} S^{*}+\mu S^{*}-\beta_{0} \varepsilon H S-\mu S\right] \\
& +b_{1}\left(1-\frac{Q^{*}}{Q}\right)\left[\beta_{0} \varepsilon H S-\frac{\beta_{0} \varepsilon H^{*} S^{*}}{Q^{*}} Q\right]+c_{1}\left(1-\frac{H^{*}}{H}\right)\left[\lambda_{q} Q-\frac{\lambda_{q} Q^{*}}{H^{*}} H\right] \\
= & -\mu a_{1} \frac{\left(S-S^{*}\right)^{2}}{S}+a_{1} \beta_{0} \varepsilon H^{*} S^{*}-\beta_{0} \varepsilon H S a_{1}-a_{1} \frac{\beta_{0} \varepsilon H^{*} S^{* 2}}{S} \\
& +a_{1} \beta_{0} \varepsilon H S^{*}+b_{1} \beta_{0} \varepsilon H S-b_{1} \beta_{0} \varepsilon \frac{H^{*} S^{*} Q}{Q^{*}}-b_{1} \beta_{0} \varepsilon \frac{H Q^{*}}{Q} \\
& +b_{1} \beta_{0} \varepsilon H^{*} S^{*}+c_{1} \lambda_{q} Q-c_{1} \lambda_{q} \frac{Q^{*} H}{H^{*}}-c_{1} \lambda_{q} \frac{Q H^{*}}{H}+c_{1} \lambda_{q} Q^{*} \\
= & -\mu a_{1} \frac{\left(S-S^{*}\right)^{2}}{S}+\beta_{0} \varepsilon H^{*} S^{*}\left(a_{1}+b_{1}\right)+\beta_{0} \varepsilon H S\left(b_{1}-a_{1}\right) \\
& +Q\left[-b_{1} \beta_{0} \varepsilon \frac{H^{*} S^{*}}{Q^{*}}+c_{1} \lambda_{q}\right]+H\left[a_{1} \beta_{0} \varepsilon S^{*}-c_{1} \lambda_{q} \frac{Q^{*}}{H^{*}}\right]-a_{1} \beta_{0} \varepsilon \frac{H^{*} S^{* 2}}{S} \\
& +c_{1} \lambda_{q} Q^{*}-b_{1} \beta_{0} \varepsilon \frac{H S Q^{*}}{Q}-c_{1} \lambda_{q} \frac{Q H^{*}}{H} .
\end{aligned}
$$

Choose the positive numbers $a_{1}, b_{1}, c_{1}$, such that

$$
\left\{\begin{array}{l}
-b_{1} \beta_{0} \varepsilon \frac{H^{*} S^{*}}{Q^{*}}+c_{1} \lambda_{q}=0 \\
a_{1} \beta_{0} \varepsilon S^{*}-c_{1} \lambda_{q} \frac{Q^{*}}{H^{*}}=0
\end{array}\right.
$$

That is for example, $a_{1}>0, c_{1}=\frac{a_{1} \beta_{0} \varepsilon S^{*} H^{*}}{\lambda_{q} Q^{*}}$ and $b_{1}=a_{1}$.

$$
\begin{aligned}
\dot{L}_{q 1}= & -\mu a_{1} \frac{\left(S-S^{*}\right)^{2}}{S}+2 a_{1} \beta_{0} \varepsilon S^{*} H^{*}+a_{1} \beta_{0} \varepsilon S^{*} H^{*} \\
& -a_{1} \beta_{0} \varepsilon \frac{H Q^{*}}{Q}-a_{1} \beta_{0} \varepsilon \frac{S^{*} Q H^{* 2}}{H Q^{*}}-a_{1} \beta_{0} \varepsilon \frac{H^{*} S^{* 2}}{S} \\
= & -\mu a_{1} \frac{\left(S-S^{*}\right)^{2}}{S}+a_{1} \beta_{0} \varepsilon S^{*} H^{*}\left(3-\frac{H S Q^{*}}{H^{*} S^{*} Q}-\frac{Q H^{*}}{H Q^{*}}-\frac{S^{*}}{S}\right) .
\end{aligned}
$$

Since $\dot{L}_{q 1}(S, Q, H)=0 \Leftrightarrow S=S^{*}, Q=Q^{*}$ and $H=H^{*}$, the largest invariant subset contained in $\left\{(S, Q, H) \in \mathbb{R}_{+}^{3} / \dot{L}_{q 1}=0\right\}$ is the unique point: $\left(S^{*}, Q^{*}, H^{*}\right)$. Hence, by LaSalle's Invariance Principle [54], this point is GAS, and so is the GAS of $E_{1}^{Q}$ of system (2.2).

Remark 3.4. The stability of the endemic equilibrium of model (2.2) suggests that the implementation of (perfect) contact tracing alone is not enough to eliminate the disease. 
In practice, contact tracing is very difficult to implement. Furthermore, if implemented, it is unlikely that all the contacts with infectives will be singled out. Therefore, one could think at the hospitalization/isolation of all infected cases as an alternative control measure to perform. Hence, the analysis of the model (2.3) below.

\subsection{Mathematical analysis of model (2.3) with perfect hospitalization: $\varepsilon_{h}=0$}

Similar to system (2.2), the well-posedness of model (2.3) is postponed to Section 3.3 as a special case of model (2.1) when $p=0$ and $Q=0$.

In what follows, the existence of equilibria for system (2.3) and their global stability are established based on the range of the corresponding control reproduction number.

Proposition 3.5. The control reproduction number of model (2.3) is

$$
\mathcal{R}_{c}^{U}=\frac{\beta_{0} \pi \lambda_{u}\left(1-p_{u s}\right)}{\mu\left(\lambda_{u}+\mu\right)\left(d_{i}+\mu+\eta_{i}\right)} .
$$

Moreover,

(i) If $\mathcal{R}_{c}^{U} \leq 1$, there exists a unique disease-free equilibrium $E_{0}^{U}$ given by $E_{0}^{U}=\left(\frac{\pi}{\mu}, 0,0,0,0\right)$.

(ii) If $\mathcal{R}_{c}^{U}>1$, there are two equilibria: the DFE and a unique endemic equilibrium $E_{1}^{U}$ whose components $\left(S_{u}^{*}, U_{u}^{*}, I_{u}^{*}, H_{u}^{*}, R_{u}^{*}\right)$ are

$$
\left\{\begin{aligned}
S_{u}^{*} & =\frac{\left(\lambda_{u}+\mu\right)\left(\eta_{i}+d_{i}+\mu\right)}{\beta_{0} \lambda_{u}\left(1-p_{u s}\right)} \\
I_{u}^{*} & =\frac{\mu\left(\lambda_{u}+\mu\right)\left[\mathcal{R}_{c}^{U}-1\right]}{\beta_{0}\left(\lambda_{u}+\mu-p_{u s} \lambda_{u}\right)} \\
U_{u}^{*} & =\frac{\mu\left(\lambda_{u}+\mu\right)\left(\eta_{i}+d_{i}+\mu\right)\left[\mathcal{R}_{c}^{U}-1\right]}{\beta_{0}\left(1-p_{u s}\right) \lambda_{u}\left(\lambda_{u}+\mu-p_{u s} \lambda_{u}\right)} \\
H_{u}^{*} & =\frac{\eta_{i} \mu\left(\lambda_{u}+\mu\right)\left[\mathcal{R}_{c}^{U}-1\right]}{\left(d_{h}+\mu+\gamma\right) \beta_{0}\left(\lambda_{u}+\mu-p_{u s} \lambda_{u}\right)} \\
R_{u}^{*} & =\frac{\gamma \eta_{i} \mu\left(\lambda_{u}+\mu\right)\left[\mathcal{R}_{c}^{U}-1\right]}{\mu\left(d_{h}+\mu+\gamma\right) \beta_{0}\left(\lambda_{u}+\mu-p_{u s} \lambda_{u}\right)}
\end{aligned}\right.
$$

Now, we can prove that the disease vanishes whenever the control reproduction number $\mathcal{R}_{c}^{U}$ is less than one, that is:

Theorem 3.6. The disease-free equilibrium $E_{0}^{U}$ of system (2.3) is GAS if and only if $\mathcal{R}_{c}^{U} \leq 1$ and is unstable if $\mathcal{R}_{c}^{U}>1$.

Proof: Consider the linear Lyapunov function candidate

$$
L_{u}=U+m I \text {, }
$$

where $m>0$ is a positive number to be determined. 
The derivative of $L_{u}$ in the direction of vector field is:

$$
L_{u}^{\prime}=U\left[-\left(\lambda_{u}+\mu\right)+\left(1-p_{u s}\right) \lambda_{u} m\right]+I\left[\beta_{0} \frac{\pi}{\mu}-\left(d_{i}+\mu+\eta_{i}\right) m\right] .
$$

We shall choose $m$ such that, $-\left(\lambda_{u}+\mu\right)+\left(1-p_{u s}\right) \lambda_{u} m=0$. That is $m=\frac{\lambda_{u}+\mu}{\left(1-p_{u s}\right) \lambda_{u}}$. Thus,

$$
\begin{aligned}
L_{u}^{\prime} & =I\left[\frac{\beta_{0} \pi}{\mu}-\frac{\left(d_{i}+\mu+\eta_{i}\right)\left(\lambda_{u}+\mu\right)}{\left(1-p_{u s}\right) \lambda_{u}}\right] \\
& =I \frac{\left(d_{i}+\mu+\eta_{i}\right)\left(\lambda_{u}+\mu\right)}{\left(1-p_{u s}\right) \lambda_{u}}\left(\mathcal{R}_{c}^{U}-1\right) \\
& \leq 0 \quad \text { if } \quad \mathcal{R}_{c}^{U} \leq 1 .
\end{aligned}
$$

Moreover, the largest invariant subset contained in the set $\left\{X \in \mathbb{R}_{+}^{5} / L_{u}^{\prime}(X)=\right.$ $0\}$ when $\mathcal{R}_{c}^{U} \leq 1$ is $\left\{E_{0}^{U}\right\}$. Thus, the conclusion follows by LaSalle's Invariance Principle [54].

As above, we shall establish the GAS of the endemic equilibrium for the special case where the untraced individuals are precisely those who had contact with infectives and will develop the disease. That is the proportion of unidentified contacts returning to the susceptible class is zero: $p_{u s}=0$. Note that $\mathcal{R}_{c}^{U}=\mathcal{R}_{c}^{U}\left(p_{u s}\right)$.

The following result is analogue of Theorem 3.16 and we shall only provide a hint for its proof.

Theorem 3.7. Assume $p_{u s}=0$, if $\mathcal{R}^{U}=\mathcal{R}_{c}^{U}(0)>1$, then the endemic equilibrium $E_{1}^{Q}$ of system (2.2) is GAS.

Proof: It suffices to use the Volterra-Lyapunov function

$$
V=\left(S-S^{*} \ln S\right)+\left(U-U^{*} \ln U\right)+\frac{\beta_{0} S^{*} I^{*}}{\lambda_{u} U^{*}}\left(I-I^{*} \ln I\right) .
$$

After analyzing the sub-models describing solely contact tracing and hospitalization separately, and due to the fact that even isolated individuals were not completely safe (during the EVD outbreak in 2014, many health workers contracted the disease by taking care of EVD patients), one could think at the investigation of a model that combines these two control strategies. This is done by analyzing the full model (2.1) below.

\subsection{Mathematical analysis of the full model (2.1)}

\subsubsection{Positivity and boundedness of the trajectories of the model}

\section{Proposition 3.8.}

$$
\text { if } \quad(S(0), Q(0), U(0), I(0), H(0), R(0)) \geq 0
$$

then, $\forall t>0, \quad(S(t), Q(t), U(t), I(t), H(t), R(t)) \geq 0$. 
Furthermore,

$$
\frac{\pi}{\mu+d_{i}+d_{h}} \leq \liminf _{t \longrightarrow+\infty} N(t) \leq \limsup _{t \longrightarrow+\infty} N(t) \leq \frac{\pi}{\mu} .
$$

Proof: The proof follows the developments in $[13,64]$.

The following result establishes the boundedness of solutions and provides a biological feasible domain for model system (3.4).

Proposition 3.9. Let $N=S+I+U+Q+R$ and

$$
\left\{\begin{array}{l}
\bar{I}=\frac{\left(1-p_{u s}\right) \lambda_{u} \pi}{\mu\left(\eta_{i}+d_{i}+\mu\right)}, \quad \bar{H}=\frac{\left(\left(1-p_{q s}\right) \lambda_{q}+\eta_{i}\right) \pi}{\mu\left(d_{h}+\mu+\gamma\right)}, \quad \bar{R}=\frac{\gamma \pi}{\mu^{2}} \\
\bar{Q}=\frac{p \beta_{0}\left(1-p_{u s}\right) \lambda_{u} \pi}{\mu\left(\lambda_{q}+\mu\right)\left(\eta_{i}+d_{i}+\mu\right)}+\frac{p \beta_{0} \varepsilon\left(\left(1-p_{q s}\right) \lambda_{q}+\eta_{i}\right) \pi}{\mu\left(\lambda_{q}+\mu\right)\left(d_{h}+\gamma+\mu\right)} \\
\bar{U}=\frac{(1-p) \beta_{0}\left(1-p_{u s}\right) \lambda_{u} \pi}{\mu\left(\lambda_{u}+\mu\right)\left(\eta_{i}+d_{i}+\mu\right)}+\frac{(1-p) \beta_{0} \varepsilon\left(\left(1-p_{q s}\right) \lambda_{q}+\eta_{i}\right) \pi}{\mu\left(\lambda_{u}+\mu\right)\left(d_{h}+\gamma+\mu\right)}
\end{array}\right.
$$

if $\quad N(0) \leq \frac{\pi}{\mu}$ then $\forall t \geq 0, N(t) \leq \frac{\pi}{\mu}, I(t) \leq \bar{I}, H(t) \leq \bar{H}, R(t) \leq \bar{R}, Q(t) \leq$ $\bar{Q}, U(t) \leq \bar{U}, \forall t \geq 0$. Moreover, the compact set $\Omega=\left\{(S(t), Q(t), U(t), I(t), H(t), R(t)) \in \mathbb{R}_{+}^{6} / N(t) \leq \frac{\pi}{\mu}\right\}$ is positively invariant.

Proof: It is straightforward that $\dot{N}=\pi-\mu N-d_{i} I-d_{h} H \leq \pi-\mu N$. Therefore, applying Gronwall lemma $[13,40,57]$, one has,

$$
\begin{gathered}
N(t) \leq \frac{\pi}{\mu}+\left(N(0)-\frac{\pi}{\mu}\right) e^{-\mu t} \quad \forall t \geq 0 . \\
\text { Thus } \quad N(t) \leq \frac{\pi}{\mu} \quad \text { if } \quad N(0) \leq \frac{\pi}{\mu} .
\end{gathered}
$$

Assume $N(0) \leq \frac{\pi}{\mu}$ then, $N(t) \leq \frac{\pi}{\mu}$. So,

$$
\dot{I}(t) \leq\left(1-p_{u s}\right) \lambda_{u} \frac{\pi}{\mu}-\left(\eta_{i}+d_{i}+\mu\right) I .
$$

Another application of Gronwall lemma yields,

$$
I(t) \leq \frac{\left(1-p_{u s}\right) \lambda_{u} \pi}{\mu\left(\eta_{i}+d_{i}+\mu\right)}=\bar{I} .
$$

The remaining inequalities are shown similarly and the positive invariance of $\Omega$ follows immediately.

As a consequence, Theorem 3.8 and Theorem 3.9 ensure the well-posedness of model (2.1) in the sense that, for any positive initial condition, there exists a unique global positive solution.

The asymptotic analysis of a large majority of epidemiological models is based on the computation of a threshold quantity called basic reproduction number. Since 
our model includes control strategies, the corresponding basic reproduction number is termed by convention control reproduction number and both of them are computed similarly [43]. Moreover, usually several epidemiological models exhibit the so-called threshold dynamics, whereby, depending on the range of the basic/control reproduction number, the existence of the disease-free and endemic equilibria are established and all the solutions of the model converge to either of them.

\subsubsection{Control reproduction number $\mathcal{R}_{c}$}

The basic reproduction number is the average number of secondary infections caused by a single infective in an entirely susceptible population during its entire infectious period $[10,33,46,50,81]$ in the absence of control measures [43]. When an infective is introduced into a susceptible population, he/she belongs either to $I, H$ or to those Ebola-deceased individuals (i.e. $\left.(1-\theta)\left(d_{i}+\mu\right) I\right)$ not safely buried who continue to transmit the disease.

- If he/she belongs to class $I$, he/she was formerly in class $U$, where he/she was introduced with the probability $(1-p)$. The average time spent in class $U$ is $\frac{1}{\lambda_{u}+\mu}$. The proportion of individuals who moved from $U$ to $I$ is $\left(1-p_{u s}\right) \lambda_{u}$. An individual of $I$ is infective during the time $\frac{1}{\eta_{i}+d_{i}+\mu}$ and have an average $\beta$ contacts with susceptibles. Hence, the contribution of individuals of class $I$ on $\mathcal{R}_{c}$ is:

$$
\frac{\beta\left(1-p_{u s}\right) \lambda_{u}(1-p)}{\left(\lambda_{u}+\mu\right)\left(\eta_{i}+d_{i}+\mu\right)} S_{0}
$$

where, $S_{0}=\frac{\pi}{\mu}$ is the number of susceptibles in the disease-free population.

- If an infected individual is not safely buried, he/she can initiate an average $\beta \nu$ contacts with susceptibles. $(1-\theta)\left(d_{i}+\mu\right) I$ is the number of EVD patients who died and are not safely buried. Of these infectives, a proportion $\frac{\left(1-p_{u s}\right) \lambda_{u}}{\lambda_{u}+\mu}$ leave compartment $U$ to $I$-compartment, where they spend on average $\frac{1}{\eta_{i}+d_{i}+\mu}$ units of time. A proportion $(1-p)$ of these infectives were susceptibles and have been in contact with infectives but still unidentified. Thus, the contribution of these individuals on $\mathcal{R}_{c}$ is:

$$
\frac{\beta \nu(1-\theta)\left(d_{i}+\mu\right)\left(1-p_{u s}\right) \lambda_{u}(1-p)}{\left(\lambda_{u}+\mu\right)\left(\eta_{i}+d_{i}+\mu\right)} S_{0}
$$

- If he/she belongs to the $H$-class, he/she may originate from the $Q$-class or the $I$ class. A Similar interpretation gives the contribution of hospitalized individuals $H$, originating from $Q$ on $\mathcal{R}_{c}$ :

$$
\frac{p\left(1-p_{q s}\right) \lambda_{q} \beta \varepsilon_{h}}{\left(\lambda_{q}+\mu\right)\left(d_{h}+\mu+\gamma\right)} S_{0}
$$


14 Berge, Ouemba, Tenkam, Lubuma

whereas, the contribution of hospitalized individuals of $H$ originating from $I$ on $\mathcal{R}_{c}$ is

$$
\frac{\eta_{i} \beta \varepsilon_{h}\left(1-p_{u s}\right) \lambda_{u}(1-p)}{\left(d_{h}+\mu+\gamma\right)\left(\eta_{i}+d_{i}+\mu\right)\left(\lambda_{u}+\mu\right)} S_{0}
$$

Altogether,

$$
\begin{aligned}
\mathcal{R}_{c}= & \frac{\beta_{0} \pi(1-p) \lambda_{u}\left(1-p_{u s}\right)}{\left(\eta_{i}+d_{i}+\mu\right)\left(\lambda_{u}+\mu\right) \mu}+\frac{\beta \varepsilon_{h} \pi \lambda_{q}\left(1-p_{q s}\right) p}{\mu\left(d_{h}+\mu+\gamma\right)\left(\lambda_{q}+\mu\right)} \\
& +\frac{\eta_{i} \beta \varepsilon_{h} \pi(1-p) \lambda_{u}\left(1-p_{u s}\right)}{\left(\eta_{i}+d_{i}+\mu\right) \mu\left(\lambda_{u}+\mu\right)\left(d_{h}+\mu+\gamma\right)}
\end{aligned}
$$

Remark 3.10. If $\varepsilon_{h}$ is small enough, the transmission caused by hospitalized individuals can be neglected. Thus, $\mathcal{R}_{c}$ for $\varepsilon_{h}=0$ is denoted by $\widetilde{\mathcal{R}_{c}}$ and satisfies

$$
\widetilde{\mathcal{R}_{c}}=\mathcal{R}_{c}\left(\varepsilon_{h}=0\right)=\frac{\beta_{0} \pi(1-p) \lambda_{u}\left(1-p_{u s}\right)}{\left(\eta_{i}+d_{i}+\mu\right)\left(\lambda_{u}+\mu\right) \mu}<\mathcal{R}_{c} .
$$

This suggests that, if a large proportion $p$ of suspected individuals is quarantined, then the disease burden will be significantly mitigated.

\subsubsection{Existence of equilibria}

Theorem 3.11. The following statements hold:

- When $\mathcal{R}_{c} \leq 1$, the model (2.1) admits only one equilibrium: the disease-free equilibrium.

- Whenever $\mathcal{R}_{c}>1$, the model (2.1) has two equilibria: namely the DFE and a unique endemic equilibrium.

Proof: Let $\left(S^{*}, Q^{*}, U^{*}, I^{*}, H^{*}, R^{*}\right)$ an equilibrium of (2.1). Setting the right hand side of (2.1) to zero and adding the first three equations gives

$$
\pi-\left(1-p_{u s}\right) \lambda_{u} U^{*}-\left(1-p_{q s}\right) \lambda_{q} Q^{*}-\mu\left(S^{*}+Q^{*}+U^{*}\right)=0 .
$$

For notational simplification, the following parameters are defined.

$$
\left\{\begin{array}{l}
\alpha_{Q}=\frac{p\left(\lambda_{u}+\mu\right)}{\left(\lambda_{q}+\mu\right)(1-p)} \\
\alpha_{I}=\frac{\left(1-p_{u s}\right) \lambda_{u}}{\eta_{i}+d_{i}+\mu} \\
\alpha_{H}=\left(\frac{\lambda_{q}\left(1-p_{q s}\right) \alpha_{Q}}{\gamma+d_{h}+\mu}+\frac{\eta_{i} \alpha_{I}}{\gamma+d_{h}+\mu}\right) \\
\alpha_{S}=\left(1-p_{u s}\right) \lambda_{u}+\left(1-p_{q s}\right) \lambda_{q} \alpha_{Q}+\mu\left(\alpha_{Q}+1\right) \\
\sigma=p_{q s} \lambda_{q} \alpha_{Q}+p_{u s} \lambda_{u}+\alpha_{S}=\frac{\lambda_{u}+\mu}{1-p}
\end{array}\right.
$$


From the second and the third equation, one can deduce

$$
\beta_{0} S^{*}\left(I^{*}+\varepsilon H^{*}\right)=\frac{\lambda_{q}+\mu}{p} Q^{*}=\frac{\lambda_{u}+\mu}{1-p} U^{*} .
$$

Thus,

$$
\left\{\begin{array}{l}
Q^{*}=\frac{p\left(\lambda_{u}+\mu\right)}{\left(\lambda_{q}+\mu\right)(1-p)} U^{*}=\alpha_{Q} U^{*}, \\
I^{*}=\frac{\left(1-p_{u s}\right) \lambda_{u} U^{*}}{\eta_{i}+d_{i}+\mu}=\alpha_{I} U^{*}, \\
H^{*}=\left(\frac{\lambda_{q}\left(1-p_{q s}\right) \alpha_{Q}}{\gamma+d_{h}+\mu}+\frac{\eta_{i} \alpha_{I}}{\gamma+d_{h}+\mu}\right) U^{*}=\alpha_{H} U^{*}, \\
R^{*}=\frac{\gamma}{\mu} \alpha_{H} U^{*} \\
S^{*}=\frac{\pi-\alpha_{S} U^{*}}{\mu} .
\end{array}\right.
$$

Since $S^{*}, Q^{*}, U^{*}, I^{*}, H^{*}$ and $R^{*}$ satisfy

$$
\pi-\beta_{0} S^{*}\left(I^{*}+\varepsilon H^{*}\right)+p_{q s} \lambda_{q} Q^{*}+p_{u s} \lambda_{u} U^{*}-\mu S^{*}=0
$$

and the fact that $\mathcal{R}_{c}$ takes the form $\mathcal{R}_{c}=\frac{\beta_{0} \pi\left(\alpha_{I}+\varepsilon \alpha_{H}\right)}{\mu \sigma}$ then by (3.8), one has

$$
U^{*}=0 \text { or } U^{*}=\frac{\mu \sigma\left(\mathcal{R}_{c}-1\right)}{\beta_{0} \alpha_{S}\left(\alpha_{I}+\varepsilon \alpha_{H}\right)}
$$

Clearly, $U^{*}=0$ refers to the DFE $E_{0}=\left(\frac{\pi}{\mu}, 0,0,0,0,0\right)$, whereas

$$
U^{*}=\frac{\mu \sigma\left(\mathcal{R}_{c}-1\right)}{\beta_{0} \alpha_{S}\left(\alpha_{I}+\varepsilon \alpha_{H}\right)}=\frac{\pi\left(\mathcal{R}_{c}-1\right)}{\alpha_{S} \mathcal{R}_{c}}
$$

gives the endemic equilibrium $E^{*}$ whose other components $S^{*}, Q^{*}, I^{*}, H^{*}, R^{*}$ are defined by (3.8).

\subsubsection{Global stability of the DFE}

The following remark is instrumental.

Remark 3.12. $\mathcal{R}_{c}$ computed above takes the form

$$
\mathcal{R}_{c}=\frac{\beta_{0} \pi\left[\frac{\left(1-p_{u s}\right) \lambda_{u}}{\eta_{i}+d_{i}+\mu}+\frac{\varepsilon\left(1-p_{q s}\right) \lambda_{q} p\left(\lambda_{u}+\mu\right)}{\left(\gamma+d_{h}+\mu\right)\left(\lambda_{q}+\mu\right)(1-p)}+\frac{\varepsilon\left(1-p_{u s}\right) \lambda_{u} \eta_{i}}{\left(\eta_{i}+d_{i}+\mu\right)\left(\gamma+d_{h}+\mu\right)}\right]}{\mu\left(p_{q s} \lambda_{q} \alpha_{Q}+p_{u s} \lambda_{u}+\alpha_{S}\right)} .
$$

Theorem 3.13. The DFE is GAS if $\mathcal{R}_{c} \leq 1$ and is unstable if $\mathcal{R}_{c}>1$. 
16 Berge, Ouemba, Tenkam, Lubuma

Proof: Consider the Lyapunov candidate function

$$
L_{0}=a Q+b U+c I+d H
$$

where, $a, b, c, d$ are positive numbers to be determined shortly.

The derivative with respect to time $t$ of $L_{0}$ is

$$
\begin{aligned}
\dot{L}_{0}= & {\left[-a\left(\lambda_{q}+\mu\right)+d\left(1-p_{q s}\right) \lambda_{q}\right] Q+\left[-b\left(\lambda_{u}+\mu\right)+c\left(1-p_{u s}\right) \lambda_{u}\right] U } \\
& +\left[\frac{p \beta_{0} a \pi}{\mu}+\frac{(1-p) \beta_{0} b \pi}{\mu}-\left(\eta_{i}+d_{i}+\mu\right) c+\eta_{i} d\right] I \\
& +\left[\frac{p \beta_{0} a \varepsilon \pi}{\mu}+\frac{(1-p) \beta_{0} b \varepsilon \pi}{\mu}-d\left(d_{h}+\mu+\gamma\right)\right] H
\end{aligned}
$$

Choose the numbers $a, b, c, d$ such that,

$$
\left\{\begin{array}{l}
-a\left(\lambda_{q}+\mu\right)+d\left(1-p_{q s}\right) \lambda_{q}=0 \\
-b\left(\lambda_{u}+\mu\right)+c\left(1-p_{u s}\right) \lambda_{u}=0 \\
\frac{p \beta_{0} a \varepsilon \pi}{\mu}+\frac{(1-p) \beta_{0} b \varepsilon \pi}{\mu}-d\left(d_{h}+\mu+\gamma\right)=0
\end{array}\right.
$$

One can take $a=1$ then,

$$
d=\frac{\lambda_{q}+\mu}{\lambda_{q}\left(1-p_{q s}\right)}, \quad b=\frac{\mu\left(d_{h}+\mu+\gamma\right)\left(\lambda_{q}+\mu\right)}{(1-p) \beta_{0} \varepsilon \pi \lambda_{q}\left(1-p_{q s}\right)}-\frac{p}{1-p}, \quad c=\frac{b\left(\lambda_{u}+\mu\right)}{\lambda_{u}\left(1-p_{u s}\right)} .
$$

We shall first prove that $b>0$ whenever $\mathcal{R}_{c} \leq 1$. By Remark 3.12, we have

$$
\begin{aligned}
\mathcal{R}_{c} \leqslant 1 & \Rightarrow \frac{\beta_{0} \pi \varepsilon\left(1-p_{q s}\right) \lambda_{q} p\left(\lambda_{u}+\mu\right)}{\left(\gamma+d_{h}+\mu\right)\left(\lambda_{q}+\mu\right)(1-p)}<\mu\left(p_{q s} \lambda_{q} \alpha_{Q}+p_{u s} \lambda_{u}+\alpha_{S}\right) \\
& \Rightarrow \frac{\frac{\beta_{0} \pi \varepsilon\left(1-p_{q s}\right) \lambda_{q} p}{\left(d_{h}+\mu+\gamma\right)\left(\lambda_{q}+\mu\right)(1-p)}}{\frac{\mu}{1-p}}<1 \\
& \Rightarrow \frac{\beta_{0} \pi \varepsilon\left(1-p_{q s}\right) \lambda_{q} p}{\mu\left(d_{h}+\mu+\gamma\right)\left(\lambda_{q}+\mu\right)(1-p)}<\frac{1}{1-p} \\
& \Rightarrow \frac{\mu\left(d_{h}+\mu+\gamma\right)\left(\lambda_{q}+\mu\right)}{\beta_{0} \pi \varepsilon\left(1-p_{q s}\right) \lambda_{q}(1-p)}>\frac{p}{1-p} \\
& \Rightarrow b>0 .
\end{aligned}
$$


Straightforward computations yield,

$$
\begin{aligned}
\dot{L}_{0}= & {\left[\frac{\left(d_{h}+\mu+\gamma\right)\left(\lambda_{q}+\mu\right)}{\varepsilon \lambda_{q}\left(1-p_{q s}\right)}+\frac{\left(\eta_{i}+d_{i}+\mu\right)\left(\lambda_{u}+\mu\right) p}{(1-p)\left(1-p_{u s}\right) \lambda_{u}}\right.} \\
& \left.-\frac{\left(\eta_{i}+d_{i}+\mu\right)\left(\lambda_{u}+\mu\right) \mu\left(d_{h}+\mu+\gamma\right)\left(\lambda_{q}+\mu\right)}{\left(1-p_{u s}\right) \lambda_{u}(1-p) \beta_{0} \varepsilon \pi \lambda_{q}\left(1-p_{q s}\right)}+\frac{\eta_{i}\left(\lambda_{q}+\mu\right)}{\lambda_{q}\left(1-p_{q s}\right)}\right] I \\
= & \frac{\left(\eta_{i}+d_{i}+\mu\right)\left(\lambda_{u}+\mu\right) \mu\left(d_{h}+\mu+\gamma\right)\left(\lambda_{q}+\mu\right)}{\left(1-p_{u s}\right) \lambda_{u}(1-p) \beta_{0} \varepsilon \pi \lambda_{q}\left(1-p_{q s}\right)}\left[\frac{\beta_{0} \pi(1-p) \lambda_{u}\left(1-p_{u s}\right)}{\left(\eta_{i}+d_{i}+\mu\right)\left(\lambda_{u}+\mu\right) \mu}\right. \\
& \left.+\frac{\beta_{0} \varepsilon \pi \lambda_{q}\left(1-p_{q s}\right) p}{\mu\left(d_{h}+\mu+\gamma\right)\left(\lambda_{q}+\mu\right)}+\frac{\eta_{i} \beta_{0} \varepsilon \pi(1-p) \lambda_{u}\left(1-p_{u s}\right)}{\left(\eta_{i}+d_{i}+\mu\right) \mu\left(\lambda_{u}+\mu\right)\left(d_{h}+\mu+\gamma\right)}-1\right] I \\
= & \frac{\left(\eta_{i}+d_{i}+\mu\right)\left(\lambda_{u}+\mu\right) \mu\left(d_{h}+\mu+\gamma\right)\left(\lambda_{q}+\mu\right)}{\left(1-p_{u s}\right) \lambda_{u}(1-p) \beta_{0} \varepsilon \pi \lambda_{q}\left(1-p_{q s}\right)}\left[\mathcal{R}_{c}-1\right] I \\
\leqslant & 0 \quad \text { if } \mathcal{R}_{c} \leqslant 1 .
\end{aligned}
$$

Now, when $\mathcal{R}_{c} \leq 1, \dot{L}_{0} \leq 0$, thus, $E_{0}$ is stable. Moreover, the largest invariant subset contained in the set $\left\{X \in \mathbb{R}_{+}^{6} / \dot{L}_{0}(X)=0\right\}$ is $\left\{E_{0}\right\}$. Thus, the conclusion follows by LaSalle's Invariance Principle [54]. When $\mathcal{R}_{c}>1, \dot{L}_{0}>0$, and $E_{0}$ is unstable.

\subsubsection{Uniform persistence of the disease}

When $\mathcal{R}_{c}>1$, the DFE is unstable and it is important to check if the disease is persistent.

Theorem 3.14. Assume $\mathcal{R}_{c}>1$, then the disease is uniformly persistent in the sense that, there exists an $\eta>0$ such that for every positive solution of (2.1), there holds:

$$
\liminf _{t \longrightarrow+\infty} Q(t)>\eta, \liminf _{t \longrightarrow+\infty} U(t)>\eta, \liminf _{t \longrightarrow+\infty} I(t)>\eta, \liminf _{t \longrightarrow+\infty} H(t)>\eta .
$$

Proof: The proof of this theorem is similar to $[39,70]$ and it suffices, after discarting the last equation of (2.1) to prove the uniform persistence of

$$
\left\{\begin{array}{l}
S^{\prime}=\pi-\beta_{0} S(I+\varepsilon H)+p_{q s} \lambda_{q} Q+p_{u s} \lambda_{u} U-\mu S \\
Q^{\prime}(t)=p \beta_{0} S(I+\varepsilon H)-\lambda_{q} Q-\mu Q \\
U^{\prime}(t)=(1-p) \beta_{0}(I+\varepsilon H)-\lambda_{u} U-\mu U \\
I^{\prime}(t)=\left(1-p_{u s}\right) \lambda_{u} U-\left(\eta_{i}+d_{i}+\mu\right) I \\
H^{\prime}(t)=\left(1-p_{q s}\right) \lambda_{q} Q+\eta_{i} I-\left(d_{h}+\mu+\gamma\right) H .
\end{array}\right.
$$

Following [70], we set

$$
\begin{aligned}
& X=\left\{(S, Q, U, I, H) \in \mathbb{R}_{+}^{5}\right\} \\
& X_{0}=\{(S, Q, U, I, H) \in X: Q, U, I, H>0\} \\
& Y=\{(S, Q, U, I, H) \in X: Q=0 \text { or } U=0 \text { or } I=0 \text { or } H=0\} .
\end{aligned}
$$


Since $Y$ contains only a single equilibrium $E_{0}$, to establish the uniform persistence of (3.9), we need to show that, $W^{s}\left(E_{0}\right) \cap X_{0}=\emptyset$, where $W^{s}\left(E_{0}\right)$ denotes the stable manifold of $E_{0}$.

Suppose this is not true, that is $W^{s}\left(E_{0}\right) \cap X_{0} \neq \emptyset$, then there exists $\left(S_{0}, Q_{0}, U_{0}, I_{0}, H_{0}\right) \in X_{0}$ such that the corresponding solution of (3.9) satisfies

$$
\lim _{t \longrightarrow+\infty}(S(t), Q(t), U(t), I(t), H(t)) \longrightarrow E_{0}=\left(\frac{\pi}{\mu}, 0,0,0,0\right) .
$$

Thus, for any $\tau>0$, there exists $T>0$ such that,

$$
\left\{\begin{array}{l}
\frac{\pi-\tau}{\mu} \leq S(t) \leq \frac{\pi+\tau}{\mu} \\
0 \leq Q(t) \leq \tau, 0 \leq U(t) \leq \tau, 0 \leq I(t) \leq \tau, 0 \leq H(t) \leq \tau, \quad \forall t \geq T,
\end{array}\right.
$$

Using Eq. (3.10) in (3.9), one has

$$
\left(\begin{array}{c}
\dot{Q(t)} \\
\dot{U(t)} \\
\dot{I(t)} \\
\dot{H(t)}
\end{array}\right) \geq J(\tau)\left(\begin{array}{c}
Q(t) \\
U(t) \\
I(t) \\
H(t)
\end{array}\right)
$$

where,

$$
J(\tau)=\left(\begin{array}{llll}
-\left(\lambda_{q}+\mu\right) & 0 & p \beta_{0} \frac{(\pi-\tau)}{\mu} & p \beta_{0} \varepsilon \frac{(\pi-\tau)}{\mu} \\
0 & -\left(\lambda_{u}+\mu\right) & (1-p) \beta_{0} \frac{(\pi-\tau)}{\mu} & (1-p) \beta_{0} \varepsilon \frac{(\pi-\tau)}{\mu} \\
0 & \left(1-p_{u s}\right) \lambda_{u}-\left(\eta_{i}+d_{i}+\mu\right) & 0 \\
\left(1-p_{q s}\right) \lambda_{q} & 0 & \eta_{i} & -\left(d_{h}+\mu+\gamma\right)
\end{array}\right) .
$$

Note that $\mathcal{R}_{c}$ given by (3.5) can be computed using the techniques in [81] by setting

$$
\mathcal{F}=\left(\begin{array}{c}
p \beta_{0} S(I+\varepsilon H) \\
(1-p) \beta_{0} S(I+\varepsilon H) \\
0 \\
0
\end{array}\right), \quad \mathcal{V}=\left(\begin{array}{c}
\left(\lambda_{q}+\mu\right) Q \\
\left(\lambda_{u}+\mu\right) U \\
-\left(1-p_{u s}\right) \lambda_{u} U+\left(\eta_{i}+d_{i}+\mu\right) I \\
-\left(1-p_{q s}\right) \lambda_{q} Q+\left(d_{h}+\mu+\gamma\right) H
\end{array}\right)
$$

so that, the Jacobian matrices $F$ and $V$ of $\mathcal{F}$ and $\mathcal{V}$ respectively at the DFE satisfy

$$
F-V=\left(\begin{array}{llll}
-\left(\lambda_{q}+\mu\right) & 0 & p \beta_{0} \frac{\pi}{\mu} & p \beta_{0} \varepsilon \frac{\pi}{\mu} \\
0 & -\left(\lambda_{u}+\mu\right) & (1-p) \beta_{0} \frac{\pi}{\mu} & (1-p) \beta_{0} \varepsilon \frac{\pi}{\mu} \\
0 & \left(1-p_{u s}\right) \lambda_{u}-\left(\eta_{i}+d_{i}+\mu\right) & 0 \\
\left(1-p_{q s}\right) \lambda_{q} 0 & \eta_{i} & -\left(d_{h}+\mu+\gamma\right)
\end{array}\right) .
$$

Let $\bar{X}=(Q, U, I, H)$ be an arbitrary solution for (3.9) and $s(A)$ the largest real part of the eigenvalues of the matrix $A$. Since $J(0)=F-V$ and $\mathcal{R}_{c}=\rho\left(F V^{-1}\right)>1$, it follows by [18] that, $s(F-V)=s(J(0))>0$. On the other hand, since $\tau>0$ is arbitrary, one can choose it small enough so that, $s(J(\tau))$ is positive. Then there 
exists a solution of the linear system $\dot{\bar{X}}=J(\tau) \bar{X}$ that grows exponentially near $\bar{X}=0$. By comparison, the solutions $\bar{X}$ of the inequation above become unbounded as $t \longrightarrow+\infty$. This is a contradiction to the fact that the solutions of system (3.9) are ultimately bounded. Therefore $W^{s}\left(E_{0}\right) \cap X_{0}=\emptyset$. Applying Theorem 4.6 in [76], one conclude that, system (2.1) is uniformly persistent with respect to $\left(X_{0}, Y\right)$.

\subsubsection{Global stability of the endemic equilibrium}

We investigate the global stability of the endemic equilibrium when contact tracing is imperfect and hospitalization is perfect. That is the same as assuming $\varepsilon=0$.

Remark 3.15. When $\varepsilon_{h}=0$, the functions $H$ and $R$ are readily given by the fifth and sixth equation. Therefore, without loss of generality the global asymptotic stability of model (2.1) is the same as that of the reduced system:

$$
\left\{\begin{array}{l}
S^{\prime}=\pi-\beta_{0} S I+p_{q s} \lambda_{q} Q+p_{u s} \lambda_{u} U-\mu S \\
Q^{\prime}=p \beta_{0} S I-\lambda_{q} Q-\mu Q \\
U^{\prime}=(1-p) \beta_{0} I-\lambda_{u} U-\mu U \\
I^{\prime}(t)=\left(1-p_{u s}\right) \lambda_{u} U-\left(\eta_{i}+d_{i}+\mu\right) I
\end{array}\right.
$$

Theorem 3.16. Let $\Omega_{Q}^{F}=\left\{(S(t), Q(t), U(t), I(t), H(t), R(t)) \in \Omega / \frac{I^{* 2}}{I^{2}}=\frac{S}{S^{*}}\right\}$. Assume $\varepsilon_{h}=0$, then the endemic equilibrium of system (2.1) is GAS in $\Omega_{Q}^{F}$ when $\mathcal{R}_{c}>1$.

Proof: Consider the Volterra-Lyapunov candidate function

$$
L_{1}=a_{1}\left(S-S^{*} \ln S\right)+a_{2}\left(Q-Q^{*} \ln Q\right)+a_{3}\left(U-U^{*} \ln U\right)+a_{4} \ln \left(I-I^{*} \ln I\right),
$$

where, $a_{i}, i=1, \ldots, 4$ are positive numbers to be determined. The time derivative of $L_{1}$ along the trajectories of system (3.11) gives:

$$
\begin{aligned}
\dot{L}_{1}= & a_{1}\left[\pi-\beta_{0} S I+p_{q s} \lambda_{q} Q+p_{u s} \lambda_{u} U-\mu S\right]\left(1-\frac{S^{*}}{S}\right) \\
& +a_{2}\left(1-\frac{Q^{*}}{Q}\right)\left[p \beta_{0} S I-\left(\lambda_{q}+\mu\right) Q\right] \\
& +a_{3}\left(1-\frac{U^{*}}{U}\right)\left[(1-p) \beta_{0} S I-\left(\lambda_{u}+\mu\right) U\right] \\
& +a_{4}\left(1-\frac{I^{*}}{I}\right)\left[\left(1-p_{u s}\right) \lambda_{u} U-\left(\eta_{i}+d_{i}+\mu\right) I\right] \\
= & a_{1}\left(1-\frac{S^{*}}{S}\right)\left[\beta_{0} S^{*} I^{*}-p_{q s} \lambda_{q} Q^{*}-p_{u s} \lambda_{u} U^{*}+\mu S^{*}-\beta_{0} S I+p_{q s} \lambda_{q} Q\right. \\
& \left.+p_{u s} \lambda_{u} U-\mu S\right]+a_{2}\left(1-\frac{Q^{*}}{Q}\right)\left[p \beta_{0} S I-p \beta_{0} S^{*} Q \frac{I^{*}}{Q^{*}}\right] \\
& +a_{3}\left(1-\frac{U^{*}}{U}\right)\left[(1-p) \beta_{0} S I-(1-p) \beta_{0} S^{*} U \frac{I^{*}}{U^{*}}\right] \\
& +a_{4}\left(1-\frac{I^{*}}{I}\right)\left[\left(1-p_{u s}\right) \lambda_{u} U-\left(1-p_{u s}\right) \lambda_{u} I \frac{U^{*}}{I^{*}}\right] .
\end{aligned}
$$


20 Berge, Ouemba, Tenkam, Lubuma

Expanding some terms yields

$$
\begin{aligned}
\dot{L}_{1}= & -\mu a_{1} \frac{\left(S-S^{*}\right)^{2}}{S}+\beta_{0} S^{*} I^{*}\left(a_{1}+p a_{2}+a_{3}(1-p)\right)-a_{4}\left(1-p_{u s}\right) \lambda_{u} \frac{U I^{*}}{I} \\
& +\beta_{0} S I\left(-a_{1}+a_{2} p+a_{3}(1-p)\right)+I\left[a_{1} \beta_{0} S^{*}-a_{4} \frac{\left(1-p_{u s}\right) \lambda_{u} U^{*}}{I^{*}}\right] \\
& +U\left[\frac{-\beta_{0} S^{*} I^{*} a_{3}(1-p)}{U^{*}}+a_{1} p_{u s} \lambda_{u}+a_{4}\left(1-p_{u s}\right) \lambda_{u}\right]-a_{2} \beta_{0} \frac{p S I Q^{*}}{Q} \\
& -a_{3}(1-p) \beta_{0} \frac{U^{*} S I}{U}-\lambda_{q} Q^{*} a_{1} p_{q s}-\frac{a_{1} p_{q s} \lambda_{q} S^{*} Q}{S}-a_{1} p_{u s} \lambda_{u} \frac{U S^{*}}{S} \\
& +\lambda_{u} U^{*}\left(-a_{1} p_{u s}+a_{4}\left(1-p_{u s}\right)\right)+Q\left[-\beta_{0} a_{2} \frac{S^{*} I^{*} p}{Q^{*}}+a_{1} p_{q s} \lambda_{q}\right] \\
& +\frac{S^{*}}{S}\left(-a_{1} \beta_{0} S^{*} I^{*}+a_{1} p_{q s} \lambda_{q} Q^{*}+a_{1} p_{u s} \lambda_{u} U^{*}\right) .
\end{aligned}
$$

Choose the $a_{i}$ such that the expressions in square brackets vanish. More precisely, $a_{1}, a_{2}, a_{3}, a_{4}$ solve the system

$$
\left\{\begin{array}{l}
-\beta_{0} a_{2} p \frac{S^{*} I^{*}}{Q^{*}}+a_{1} p_{q s} \lambda_{q}=0 \\
-\beta_{0} a_{3}(1-p) \frac{S^{*} I^{*}}{U^{*}}+a_{1} p_{u s} \lambda_{u}+a_{4}\left(1-p_{u s}\right) \lambda_{u}=0 \\
a_{1} \beta_{0} S^{*}-a_{4}\left(1-p_{u s}\right) \lambda_{u} \frac{U^{*}}{I^{*}}=0
\end{array}\right.
$$

whose solution is

$$
a_{2}=\frac{a_{1} p_{q s} \lambda_{q} Q^{*}}{p \beta_{0} S^{*} I^{*}}, a_{3}=\frac{a_{1} U^{*} p_{u s} \lambda_{u}+a_{1} \beta_{0} S^{*} I^{*}}{\beta_{0} S^{*}(1-p) I^{*}}, a_{4}=\frac{a_{1} \beta_{0} S^{*} I^{*}}{\left(1-p_{u s}\right) \lambda_{u} U^{*}} .
$$

Straightforward computations yield

$$
\begin{aligned}
\dot{L}_{1}= & -\mu a_{1} \frac{\left(S-S^{*}\right)^{2}}{S}+a_{1} \beta_{0} S^{*} I^{*}+a_{1} p_{q s} \lambda_{q} Q^{*}+a_{1} U^{*} p_{u s} \lambda_{u}+2 a_{1} \beta_{0} S^{*} I^{*} \\
& -\beta_{0} S I a_{1}+p_{q s} \lambda_{q} a_{1} Q^{*} \frac{S I}{S^{*} I^{*}}+a_{1} p_{u s} \lambda_{u} U^{*} \frac{S I}{S^{*} I^{*}}+a_{1} \beta_{0} S I-a_{1} p_{q s} \lambda_{q} \frac{S I Q^{* 2}}{Q S^{*} I^{*}} \\
& -a_{1} p_{u s} \lambda_{u} \frac{S I U^{* 2}}{S^{*} I^{*} U}-a_{1} \beta_{0} \frac{U^{*} S I}{U}-a_{1} p_{q s} \lambda_{q} Q^{*}-a_{1} p_{q s} \lambda_{q} \frac{S^{*} Q}{S}-a_{1} p_{u s} \lambda_{u} U^{*} \\
& -a_{1} p_{u s} \lambda_{u} \frac{U S^{*}}{S}-a_{1} \beta_{0} \frac{U I^{* 2} S^{*}}{U^{*} I}+a_{1} p_{q s} \lambda_{q} \frac{Q^{*} S^{*}}{S}-a_{1} \beta_{0} \frac{S^{* 2} I^{*}}{S}+a_{1} p_{u s} \lambda_{u} \frac{U^{*} S^{*}}{S} .
\end{aligned}
$$


Denote $\varphi\left(S^{*}\right)=\pi-\mu S^{*}=\beta_{0} S^{*} I^{*}-p_{q s} \lambda_{q} Q^{*}-p_{u s} \lambda_{u} U^{*}$. Then

$$
\begin{aligned}
& \dot{L}_{1}=-\mu a_{1} \frac{\left(S-S^{*}\right)^{2}}{S}+a_{1} \beta_{0} S^{*} I^{*}\left(3-\frac{S^{*}}{S}-\frac{U I^{*}}{U^{*} I}-\frac{I S U^{*}}{U I^{*} S^{*}}\right) \\
& +a_{1} \frac{S^{*}}{S}\left(p_{q s} \lambda_{q} Q^{*}+p_{u s} \lambda_{u} U^{*}\right)-a_{1} p_{q s} \lambda_{q} \frac{S I Q^{* 2}}{Q S^{*} I^{*}}-a_{1} p_{u s} \lambda_{u} \frac{S I U^{* 2}}{S^{*} I^{*} U} \\
& -a_{1} p_{u s} \lambda_{u} \frac{U S^{*}}{S}-a_{1} p_{q s} \lambda_{q} \frac{S^{*} Q}{S}+a_{1} \frac{S I}{S^{*} I^{*}}\left(p_{q s} \lambda_{q} Q^{*}+p_{u s} \lambda_{u} U^{*}\right) \\
& =-\mu a_{1} \frac{\left(S-S^{*}\right)^{2}}{S}+a_{1} \beta_{0} S^{*} I^{*}\left(3-\frac{S^{*}}{S}-\frac{U I^{*}}{U^{*} I}-\frac{I S U^{*}}{U I^{*} S^{*}}\right) \\
& +a_{1} \frac{S^{*}}{S}\left(\beta_{0} S^{*} I^{*}-\varphi\left(S^{*}\right)\right)-a_{1} p_{q s} \lambda_{q} \frac{S I Q^{* 2}}{Q S^{*} I^{*}}-a_{1} p_{u s} \lambda_{u} \frac{S I U^{* 2}}{S^{*} I^{*} U} \\
& -a_{1} p_{q s} \lambda_{q} \frac{S^{*} Q}{S}-a_{1} p_{u s} \lambda_{u} \frac{U S^{*}}{S}+a_{1} \frac{S I}{S^{*} I^{*}}\left(\beta_{0} S^{*} I^{*}-\varphi\left(S^{*}\right)\right) \\
& =-\mu a_{1} \frac{\left(S-S^{*}\right)^{2}}{S}+a_{1} \beta_{0} S^{*} I^{*}\left(3-\frac{S^{*}}{S}-\frac{U I^{*}}{U^{*} I}-\frac{I S U^{*}}{U I^{*} S^{*}}\right)-a_{1} p_{u s} \lambda_{u} \frac{S I U^{* 2}}{S^{*} I^{*} U} \\
& -3 a_{1} \varphi\left(S^{*}\right)+a_{1} \varphi\left(S^{*}\right) \frac{I^{*}}{I}+a_{1} \beta_{0} S I+a_{1} \beta_{0} \frac{S^{* 2} I^{*}}{S}-a_{1} p_{q s} \lambda_{q} \frac{S I Q^{* 2}}{Q S^{*} I^{*}} \\
& -a_{1} p_{q s} \lambda_{q} \frac{S^{*} Q}{S}-a_{1} p_{u s} \lambda_{u} \frac{U S^{*}}{S}+a_{1} \varphi\left(S^{*}\right)\left(3-\frac{S^{*}}{S}-\frac{S I}{S^{*} I^{*}}-\frac{I^{*}}{I}\right) \\
& =-\mu a_{1} \frac{\left(S-S^{*}\right)^{2}}{S}+a_{1} \beta_{0} S^{*} I^{*}\left(3-\frac{S^{*}}{S}-\frac{U I^{*}}{U^{*} I}-\frac{I S U^{*}}{U I^{*} S^{*}}\right)-3 a_{1} \beta_{0} S^{*} I^{*} \\
& +a_{1} \varphi\left(S^{*}\right)\left(3-\frac{S^{*}}{S}-\frac{S I}{S^{*} I^{*}}-\frac{I^{*}}{I}\right)+3 p_{q s} \lambda_{q} Q^{*} a_{1}+3 p_{u s} \lambda_{u} U^{*} a_{1} \\
& +a_{1} \beta_{0} \frac{S^{*} I^{* 2}}{I}-p_{q s} \lambda_{q} a_{1} \frac{Q^{*} I^{*}}{I}-p_{u s} \lambda_{u} a_{1} \frac{U^{*} I^{*}}{I}+a_{1} \beta_{0} S I+a_{1} \beta_{0} \frac{S^{* 2} I^{*}}{S} \\
& -a_{1} p_{q s} \lambda_{q} \frac{S I Q^{* 2}}{Q S^{*} I^{*}}-a_{1} p_{u s} \lambda_{u} \frac{S I U^{* 2}}{S^{*} I^{*} U}-a_{1} p_{q s} \lambda_{q} \frac{S^{*} Q}{S}-a_{1} p_{u s} \lambda_{u} \frac{U S^{*}}{S} \\
& =-\mu a_{1} \frac{\left(S-S^{*}\right)^{2}}{S}+a_{1} \beta_{0} S^{*} I^{*}\left(3-\frac{S^{*}}{S}-\frac{U I^{*}}{U^{*} I}-\frac{I S U^{*}}{U I^{*} S^{*}}\right) \\
& +a_{1} \varphi\left(S^{*}\right)\left(3-\frac{S^{*}}{S}-\frac{S I}{S^{*} I^{*}}-\frac{I^{*}}{I}\right)-a_{1} \beta_{0} S^{*} I^{*}\left(3-\frac{I^{*}}{I}-\frac{S I}{S^{*} I^{*}}-\frac{S^{*}}{S}\right) \\
& +a_{1} p_{q s} \lambda_{q} Q^{*}\left(3-\frac{I^{*}}{I}-\frac{S I Q^{*}}{S^{*} I^{*} Q}-\frac{Q S^{*}}{Q^{*} S}\right) \\
& +a_{1} p_{u s} \lambda_{u} U^{*}\left(3-\frac{I^{*}}{I}-\frac{S I U^{*}}{S^{*} I^{*} U}-\frac{U S^{*}}{U^{*} S}\right)
\end{aligned}
$$




$$
\begin{aligned}
= & -\mu a_{1} \frac{\left(S-S^{*}\right)^{2}}{S}+a_{1} \varphi\left(S^{*}\right)\left(3-\frac{S^{*}}{S}-\frac{S I}{S^{*} I^{*}}-\frac{I^{*}}{I}\right) \\
& +a_{1} p_{q s} \lambda_{q} Q^{*}\left(3-\frac{I^{*}}{I}-\frac{S I Q^{*}}{S^{*} I^{*} Q}-\frac{Q S^{*}}{Q^{*} S}\right) \\
& +a_{1} p_{u s} \lambda_{u} U^{*}\left(3-\frac{I^{*}}{I}-\frac{S I U^{*}}{S^{*} I^{*} U}-\frac{U S^{*}}{U^{*} S}\right) \\
& +a_{1} \beta_{0} S^{*} I^{*}\left(3-\frac{S^{*}}{S}-\frac{U I^{*}}{U^{*} I}-\frac{I S U^{*}}{U I^{*} S^{*}}\right)-a_{1} \beta_{0} S^{*} I^{*}\left(3-\frac{I^{*}}{I}-\frac{S I}{S^{*} I^{*}}-\frac{S^{*}}{S}\right) \\
= & -\mu a_{1} \frac{\left(S-S^{*}\right)^{2}}{S}+a_{1} \varphi\left(S^{*}\right)\left(3-\frac{S^{*}}{S}-\frac{S I}{S^{*} I^{*}}-\frac{I^{*}}{I}\right) \\
& +a_{1} p_{q s} \lambda_{q} Q^{*}\left(3-\frac{I^{*}}{I}-\frac{S I Q^{*}}{S^{*} I^{*} Q}-\frac{Q S^{*}}{Q^{*} S}\right) \\
& +a_{1} p_{u s} \lambda_{u} U^{*}\left(3-\frac{I^{*}}{I}-\frac{S I U^{*}}{S^{*} I^{*} U}-\frac{U S^{*}}{U^{*} S}\right) \\
& +a_{1} \beta_{0} S^{*} I^{*}\left(\frac{I^{*}}{I}+\frac{S I}{S^{*} I^{*}}-\frac{U I^{*}}{U^{*} I}-\frac{I S U^{*}}{U I^{*} S^{*}}\right) \\
= & -\mu a_{1} \frac{\left(S-S^{*}\right)^{2}}{S}+a_{1} \varphi\left(S^{*}\right)\left(3-\frac{S^{*}}{S}-\frac{S I}{S^{*} I^{*}}-\frac{I^{*}}{I}\right) \\
& +a_{1} p_{q s} \lambda_{q} Q^{*}\left(3-\frac{I^{*}}{I}-\frac{S I Q^{*}}{S^{*} I^{*} Q}-\frac{Q S^{*}}{Q^{*} S}\right) \\
& +a_{1} p_{u s} \lambda_{u} U^{*}\left(3-\frac{I^{*}}{I}-\frac{S I U^{*}}{S^{*} I^{*} U}-\frac{U S^{*}}{U^{*} S}\right) \\
& +a_{1} \beta_{0} S^{*} I^{*}\left[\frac{I^{*}}{I}\left(1-\frac{U}{U^{*}}\right)+\frac{S I}{S^{*} I^{*}}\left(1-\frac{U^{*}}{U}\right)\right]
\end{aligned}
$$

Since we are working in $\Omega_{Q}^{F}$, we have $\frac{I^{* 2}}{I^{2}}=\frac{S}{S^{*}}$. Thus, $\frac{I^{*}}{I}=\frac{S I}{S^{*} I^{*}}$ and $\dot{L}_{1}$ becomes

$$
\begin{aligned}
\dot{L}_{1}= & -\mu a_{1} \frac{\left(S-S^{*}\right)^{2}}{S}+a_{1} \varphi\left(S^{*}\right)\left(3-\frac{S^{*}}{S}-\frac{S I}{S^{*} I^{*}}-\frac{I^{*}}{I}\right) \\
& +a_{1} p_{q s} \lambda_{q} Q^{*}\left(3-\frac{I^{*}}{I}-\frac{S I Q^{*}}{S^{*} I^{*} Q}-\frac{Q S^{*}}{Q^{*} S}\right) \\
& +a_{1} p_{u s} \lambda_{u} U^{*}\left(3-\frac{I^{*}}{I}-\frac{S I U^{*}}{S^{*} I^{*} U}-\frac{U S^{*}}{U^{*} S}\right) \\
& +\frac{a_{1} \beta_{0} S^{*} I^{* 2}}{I}\left(2-\frac{U}{U^{*}}-\frac{U^{*}}{U}\right) . \\
\dot{L}_{1} \leq & 0 .
\end{aligned}
$$

Moreover $\dot{L}_{1}=0$ if and only if $S=S^{*}, U=U^{*}, Q=Q^{*}$ and $I=I^{*}$ so that the largest invariant set contained in $\left\{(S, Q, U, I) \in \mathbb{R}_{+}^{4} / \dot{L}_{1}=0\right\}$ is the unique endemic point $E^{*}=\left(S^{*}, Q^{*}, U^{*}, I^{*}\right)$. Hence, by LaSalle's Invariance Principle [54], $E^{*}$ is GAS in $\Omega_{Q}^{F}$.

Remark 3.17. The following features are worth mentioning.

- Theorem 3.16 states that, in spite of a perfect isolation/hospitalization, EVD can remain endemic if the contacts are not fully traced. 
- If $p_{q s}=p_{u s}=0$, i.e neither traced nor untraced individuals move back to the susceptible class, then the condition $\frac{I^{* 2}}{I^{2}}=\frac{S}{S^{*}}$ can be relaxed such that the endemic equilibrium of model (3.4) is GAS in the entiere $\Omega$.

- The assumption $\varepsilon_{h}=0$ means that, healthcare workers use adequate equipments to protect themselves from contracting EVD.

It is worth noticeable that, in the case of (i) perfect contact tracing only (model $(2.2)$ ), (ii) hospitalization/isolation only (model (2.3)), and (iii) imperfect contact tracing and hospitalization (full model (2.1)), we have shown that, when the control reproduction number is less than one, the disease dies out. So, the challenge is to bring $\mathcal{R}_{c}$ below one. Since $\mathcal{R}_{c}$ depends on many parameters, it is therefore meaningful to select those which are most influential. This is where the local or global sensitivity analysis comes in $[52,58]$.

\section{Sensitivity analysis of the control reproduction number}

\subsection{Parameters estimation}

The majority of parameters in Table 2 are mainly obtained from $[47,72,80]$. In addition, $\lambda_{u}, \lambda_{q}, p_{q s}, p_{u s}, \theta$, are estimated according to $[1,2,22,73,75]$. Precisely:

- Since individuals in compartments $Q$ and $U$ can be regarded as latently infected people, $1 / \lambda_{u}$ and $1 / \lambda_{q}$ can be seen as the duration of latency which is generally between 8 and 10 days $[1,2,4,75]$. We simply choose $1 / \lambda_{u}$ to be the minimum latent period and $1 / \lambda_{q}$ to be its maximum.

- $p_{q s}$ can be interpreted as the probability for a susceptible to remain uninfected after exposure to EVD. It is documented that, from one infected in Nigeria, a total of 894 contacts were identified amongst which 19 have contracted the virus $[74,75]$. Therefore, $894-19=875$ contacts were uninfected. Thus, the probability for a susceptible to remain uninfected after exposure to EVD can be estimated by $p_{q s}=875 / 894 \approx 0.978$. For the sake of simplicity, we assume that $p_{u s}=p_{q s}$.

- Following [73], it is known that $\theta$ lies in $[0.33,7]$ per week. In this work, $\theta$ is chosen to be the mean between the minimum and the maximum values per day. Thus, $\theta=\frac{1}{2}\left(\frac{0.33+7}{7}\right)=0.523$.

\subsection{Computation of the forward normalized sensitivity indices}

We perform sensitivity analysis to identify most influential parameters on $\mathcal{R}_{c}$. Actually, sensitivity analysis is very important in designing control strategies [52,58]. The change in the value of $\mathcal{R}_{c}$ can be measured using the forward normalized sensitivity index of $\mathcal{R}_{c}$ with respect to the model parameter $\alpha$ and is defined by $[21,52,75]$ :

$$
\varepsilon_{\alpha}^{\mathcal{R}_{c}}=\frac{\partial \mathcal{R}_{c}}{\partial \alpha} \cdot \frac{\alpha}{\mathcal{R}_{c}} .
$$


24 Berge, Ouemba, Tenkam, Lubuma

\begin{tabular}{|llll|}
\hline Parameter & Baseline value & Source & Sensitivity index \\
\hline$\beta$ & 0.16 & {$[72]$} & 1 \\
$p$ & 0.3 & {$[80]$} & -0.251 \\
$\nu$ & $0.111 / 0.16$ & {$[72]$} & 0.162 \\
$\varepsilon_{h}$ & $0.062 / 0.16$ & {$[72]$} & 0.196 \\
$\pi$ & 2.5 & {$[47]$} & 1 \\
$d_{i}$ & 0.75 & {$[80]$} & $-0,492$ \\
$d_{h}$ & 0.3 & {$[80]$} & -0.069 \\
$\gamma$ & 0.5366 & {$[42]$} & -0.1256 \\
$\theta$ & 0.523 & Estimated & -0.177 \\
$\lambda_{q}$ & $1 / 10$ & Estimated & 0.113 \\
$p_{q s}$ & 0.978 & Estimated & -5.51 \\
$\lambda_{u}$ & $1 / 8$ & Estimated & 0.064 \\
$p_{u s}$ & 0.978 & Estimated & -38.94 \\
$\mu$ & $10.17 / 1000$ & {$[10]$} & -1.09 \\
$\eta_{i}$ & 0.25 & {$[47]$} & -0.144 \\
\hline
\end{tabular}

Table 2. Sensitivity index of $\mathcal{R}_{c}$ : parameter values for simulations.

Note that $\varepsilon_{\alpha}^{\mathcal{R}_{c}}$ is positive if $\mathcal{R}_{c}$ is increasing with respect to $\alpha$, and negative if $\mathcal{R}_{c}$ is decreasing with respect to $\alpha$. The sensitive indices of $\mathcal{R}_{c}$ at the baseline values of model (2.1) are displayed in Table 2.

This sensitivity analysis shows that $\mathcal{R}_{c}$ decreases when $p, \eta_{i}, p_{u s}$ and $p_{q s}$ increase. In particular, $p_{u s}$ and $p_{q s}$ are the most influential parameters on $\mathcal{R}_{c}$. This means that: the smaller the probability of remaining susceptible after contact (identified or not) with infectives, the bigger the control reproduction number and the lower the chance to control EVD. This detrimental feature can be avoided by using protective clothes while taking care of infectives. Moreover, $\mathcal{R}_{c}$ increases fastly with the parameters $\beta, \varepsilon_{h}$ and $\pi$. One way of reducing $\beta$ and $\varepsilon_{h}$ is to educate people so that susceptible individuals can easily recognize EVD related symptoms and send an alert to health authorities for proper management of the disease [20]. As for $\pi$, one way of lowering it is to avoid immigration by shutting down the borders of the affected region. Conversely, as mentioned earlier, a good implementation of contact tracing can increase the quarantine rate $p$ and the hospitalization rate $\eta_{i}$ leading to a decrease in $\mathcal{R}_{c}$ and a greater chance to eradicate EVD.

\subsection{Impacts of $p$ and $\varepsilon_{h}$ on the control reproduction number}

Although, according to Table 2, $p$ and $\varepsilon_{h}$ are not the most sensitive parameters for $\mathcal{R}_{c}$, it is worth noticing from Remark 3.10 that, these latter quantities can represent some key control parameters in order to mitigate EVD. Therefore, one 
may wish to observe how $\mathcal{R}_{c}$ behaves as a function of $p$ and $\eta_{i}$. This is shown in Figure 2 below, and it is not surprising (see the above subsection) to realise that $\mathcal{R}_{c}$ decreases when $p$ increases and increases when $\varepsilon_{h}$ increases (consistency with Table 2). Additionally, Figure 2 depicts that $p$ have a higher influence on $\mathcal{R}_{c}$ (by lowering it faster) than $\varepsilon_{h}$. Since $p$ and $\varepsilon_{h}$ represent the efficacy of contact tracing and isolation, respectively, the latter remark suggests that contact tracing is a better control measure than isolation, at least at the onset of the disease outbreak. We

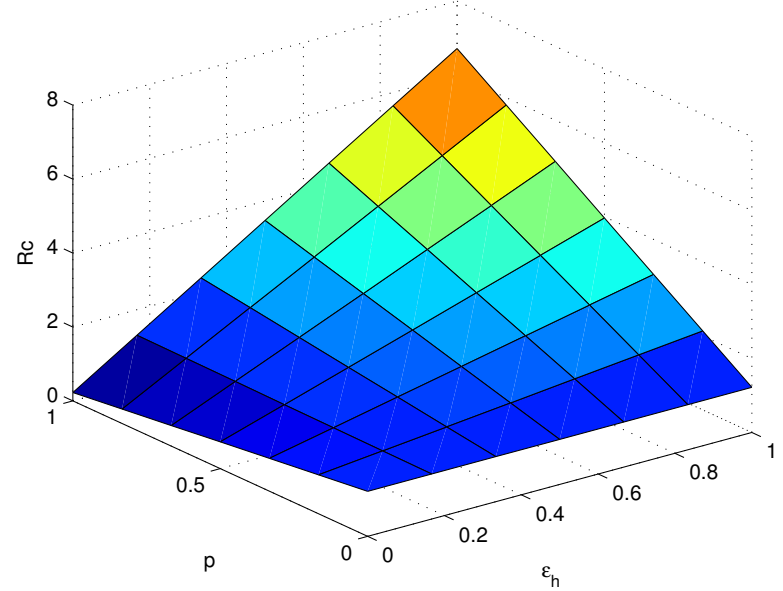

Fig. 2. Control reproduction number in function of $\varepsilon_{h}$ and $p$ (the other parameters are in Table 2).

have shown from the sensitivity analysis in Table 2 that $p$ and $\varepsilon_{h}$ do not greatly influence the disease outbreak estimate $\mathcal{R}_{c}$. However, in what follows, are going to demonstrate numerically that these two parameters can highly impact the long run of the disease.

\section{Numerical simulations}

As before, simulations are performed under MatLab using ODE 45 in order to numerically illustrate the impact of contact tracing and isolation on the long run dynamics of EVD. We will simulate our full model with most of the baseline parameters drawn from Table 2.

\subsection{Global asymptotic stability of equilibria}

The GAS of the disease-free equilibrium and the local asymptotic stability of the endemic equilibrium (in the case of a full hospitalization) of model (2.1) were analytically shown in Theorem 3.13 and Theorem 3.16, respectively. Here, they are numerically supported by Figure 3 and Figure 4 which are plotted for $\beta=0.16, p=0.8$ 
(Figure 3 ) and $\beta=0.3, p=0.1$ (Figure 4). Precisely, Figure 3 suggests that, when $\mathcal{R}_{c}=0.6913$, EVD is eradicated after approximately 310 days, while Figure 4 suggests that, for $\mathcal{R}_{c}=2.4759$, EVD persists and becomes endemic. Moreover these figures illustrate that infected individuals $I(t)$ and $H(t)$ decrease when both (imperfect) contact tracing and isolation are implemented.

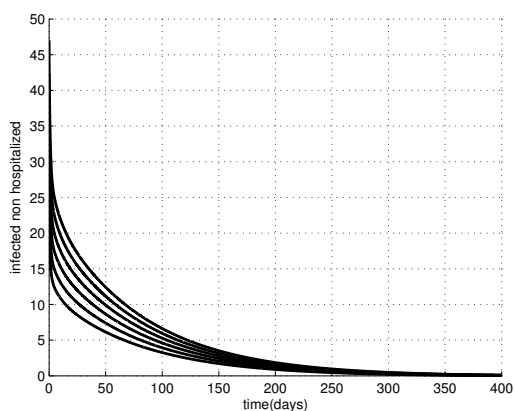

(a)

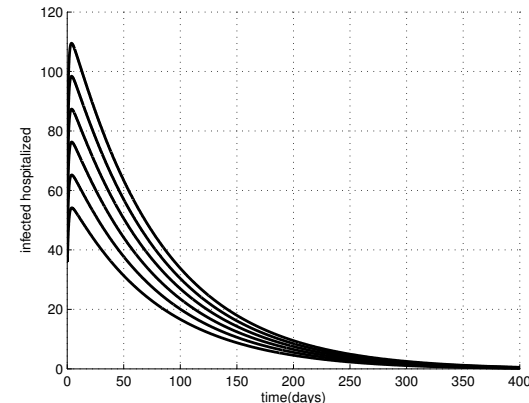

(b)

Fig. 3. GAS of the disease-free equilibrium when $\mathcal{R}_{c}=0.6913<1$. The solution curves (a) and (b) are plotted with $\beta=0.16, p=0.8$ and the remaining parameters as in Table 2

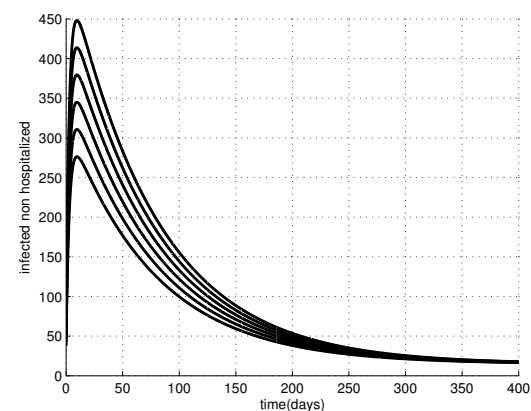

(a)

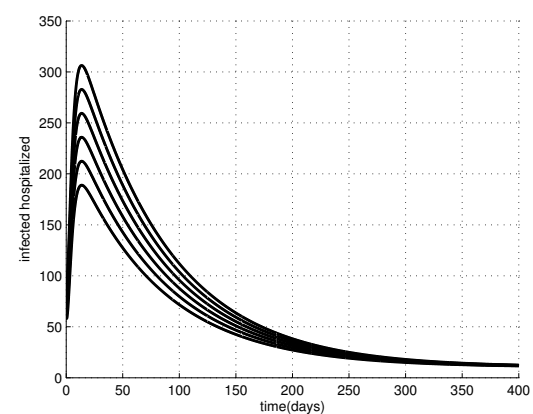

(b)

Fig. 4. GAS of the endemic equilibrium when $\mathcal{R}_{c}=2.4759>1$. The solution curves (a) and (b) are plotted with $\beta=0.3, p=0.1$ and the remaining parameters as in Table 2)

\subsection{Assessing the efficiency of contact tracing}

Without loss of generality, we assume that contact tracing acts only on parameter $p$. Figure 5 shows that infected (non-hospitalized) individuals $I(t)$ is a decreasing function of the efficacy parameter $p$ of contact tracing. More precisely, when there is no contact tracing at all $(p=0)$, during the first two days, the infected number increases very fast. After 117 infected, this number decreases but the disease persists 
in the community (recall $\mathcal{R}_{c}=1.0820$ in this case). If contact tracing is boosted up to $80 \%(p=0.8)$, then the disease drops down immediately and goes to extinction: $\mathcal{R}_{c}<1$. So, a high rate implementation of contact tracing, by identifying and quarantining most of the contacts (not necessary all) can mitigate the infection.

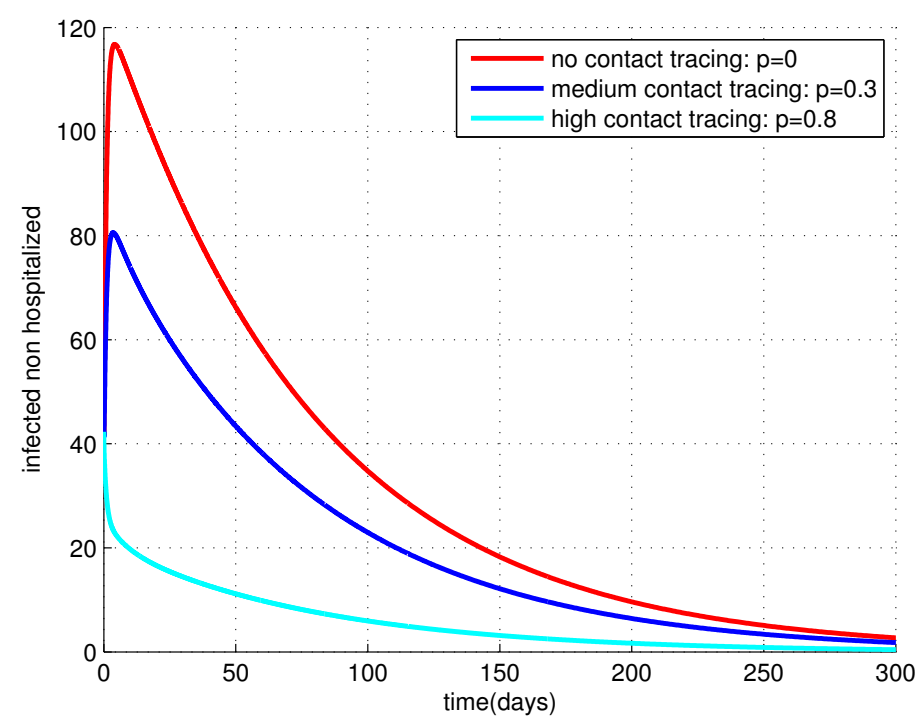

Fig. 5. Efficiency of contact tracing: $I(t)$ as a function of the contact tracing rate $p$. $\mathcal{R}_{c}=1.0820(p=0), \mathcal{R}_{c}=0.6913(p=0.03)$ and $\mathcal{R}_{c}=0.4027(p=0.8), \varepsilon_{h}=0.38$.

\subsection{Impact of hospitalization/isolation}

Figure 5 is plotted for a small value of $\varepsilon_{h}\left(\varepsilon_{h}=0.38\right)$. Having in mind that $\varepsilon_{h}$ measures the efficacy of hospitalization/isolation, and due to the fact that in Africa, people usually take care of their relatives at the hospital, one may think of assessing the success of perfect isolation, by taking $\varepsilon_{h}=0$. On the other hand, one can worsen isolation (from $\varepsilon_{h}=0.38$ to $\varepsilon_{h}=0.8$ ) and pair it with a medium contact tracing $(p=0.3)$ in order to compare this combined control strategy with perfect isolation $\left(\varepsilon_{h}=0\right)$ and no contact tracing. Figure 6 shows that both strategies act positively at the early stage (approximatively 10 days) by decreasing the number of infectives $I(t)$. However, one can notice that up to 260 days, the pairing of medium contact tracing and poor isolation (red curve) is better than perfect isolation alone (blue curve) and that the situation is reversed after. Moreover, looking closely at the zoomed Figure 6(b), we realise that perfect isolation alone (blue curve) reduces sufficiently $I(t)$ and drives it to extinction. Hence the favorable impact of isolation on the reduction of the epidemic level, as well as on the mitigation of the disease. 

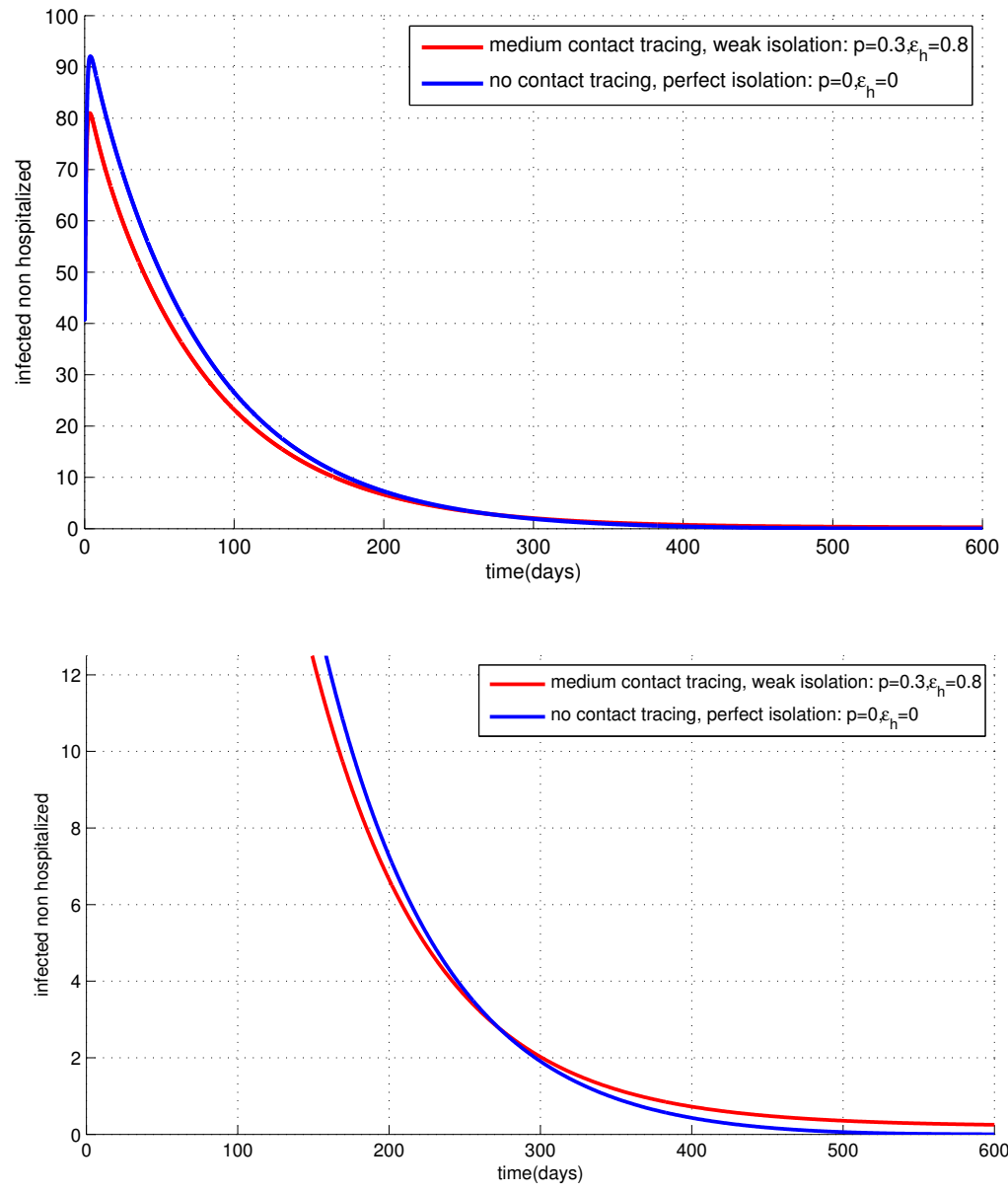

(b)

Fig. 6. Efficacy of isolation: $I(t)$ as a function of $\varepsilon_{h}: p=0, \varepsilon_{h}=0$ (blue) and $p=0.3, \varepsilon_{h}=0.8$ (red)

\subsection{Contact tracing versus isolation}

We have shown in the previous subsections that either (i)-high contact tracing or (ii)-medium contact tracing with medium isolation or (iii)-perfect isolation without contact tracing, can mitigate EVD. However, it would be better if we can single out the best control strategy among them. An attempt to answer this question can be provided by plotting the solution of the total number of infected individuals $(I(t)+H(t))$ in the case of (1)-high contact tracing and perfect isolation $(p=$ $\left.0.9, \varepsilon_{h}=0\right)$, (2)-perfect contact tracing without isolation $\left(p=1, \varepsilon_{h}=1\right)$, (3)medium contact tracing with medium isolation $\left(p=0.3, \varepsilon_{h}=0.3875\right)$, and (4)perfect isolation no contact tracing $\left(p=0, \varepsilon_{h}=0\right)$. Figure 7 suggests clearly that, strategy (1) is the best in the sense that, this control measure leads to less infected 
individuals compared to strategies (2), (3), and (4). Hence, a combination of high contact tracing (up to $90 \%$ ) and a full hospitalization/perfect isolation can be the panacea to fight against EVD.

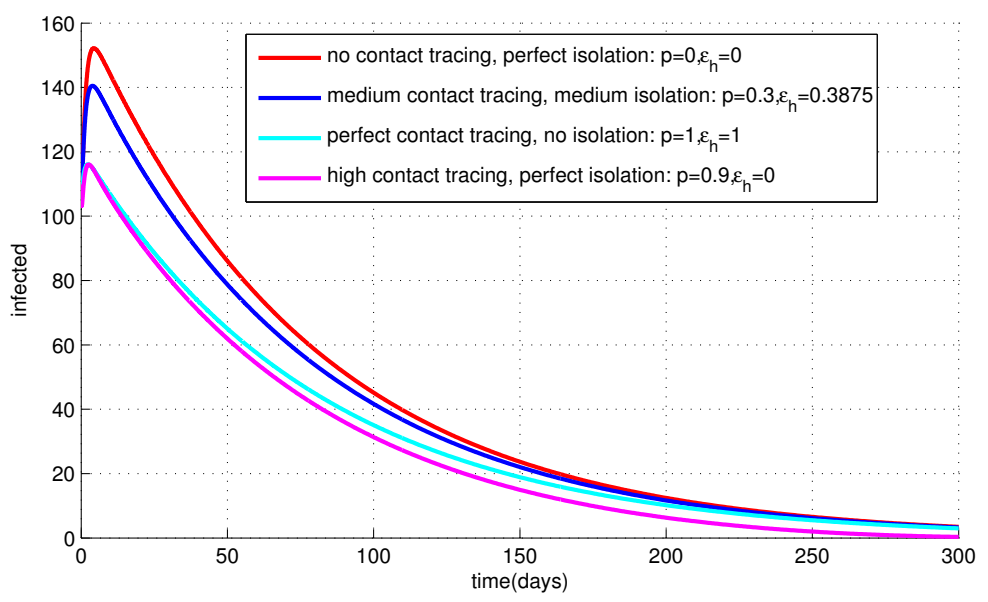

Fig. 7. Total number of infected (hospitalized and non-hospitalized) and recovered in the case of:

(i) effective isolation without contact tracing (red curve),

(ii) medium contact tracing and medium isolation (blue curve),

(iii) perfect contact tracing without isolation at all (cyan curve), and

(iv) high contact tracing and effective isolation (full hospitalization): $p=0.9, \varepsilon_{h}=0$ (magenta curve) .

\section{Discussion and perspectives}

Many mathematical epidemics models were proposed and analyzed to explain human-human transmission of EVD, but very few considered contact tracing and assessed its impact on the control of the disease $[42,71,72]$. In this paper, we focused mainly on this strategy (which implies quarantine and isolation/hospitalization), bearing in our mind that its implementation does not always lead to the identification of all contacts. Additionally, our generic model (2.1) incorporated the following three aspects: (i) the probable infected cases (i.e individuals who had contacts with infectives) were quarantined. They were gathered in the $Q$-compartment and underwent supportive treatment immediately in the case they have exhibited EVD symptoms, (ii) the hospitalized individuals were assembled into the $H$-compartment, and we assumed that they were less likely to infect susceptibles individuals (class $S$ ), (iii) the infected (non-hospitalized) individuals in the $(I)$-class who died out of the hospital could transmit infection during funerals.

On the one hand, a qualitative analysis of the full model (2.1) and the derived sub-models as well as a sensitivity analysis of the control reproduction number were investigated. In this regard, the main results obtained are summarized as follows: 
- Regardless the model/sub-model considered, when the corresponding control reproduction number is less than one, the DFE is GAS. Epidemiologically, this means that, irrespective of the initial number of infected individuals, if the control reproduction number is below one, the disease will asymptotically drives to extinction. Conversely, when the control reproduction number is greater than one, the DFE becomes unstable, the disease is persists and a GAS endemic equilibrium occurs.

- The control reproduction number is more sensitive to the contact tracing strategy than the isolation strategy.

- The probabilities of remaining uninfected after exposure to suspected individuals $\left(p_{q s}, p_{u s}\right)$ have the highest impacts on the control reproduction number.

On the other hand, numerically, we have shown that: (1) the disease can be mitigated if a great proportion of contacts are identified (high value of $p$ ) and only few of them are infected (small value of $p_{q s}$ ). (2) the number of infected individuals decreases when the contact tracing increases. (3) The combination of high contact tracing and perfect isolation leads to less infected individuals compared to all other control measures namely: (i) perfect contact tracing, (ii) both medium contact tracing and isolation, (iii) full hospitalization/isolation without contact tracing at all.

The above mentioned theoretical and numerical studies suggest that: (a) Perfect contact tracing alone is not sufficient to drive EVD to death, specially if the transmission is sustained in the hospital. (b) Perfect hospitalization alone is not enough to control EVD.

Therefore, fighting against EVD by tracing contacts requires: quarantining a great number of persons who had contacts with infectives, reducing the contacts between susceptibles with hospitalized/isolated and the wearing of protective clothes before taking care of EVD patients.

Our model has some limitations. Firstly, we have assumed that the identification of a contact is instantaneous, it would be more realistic to account for the time lag between a contact and its identification. Secondly, due to the unavailability of some parameters, many of them have been estimated and might have been over/underestimated. Thirdly, since it is well known and documented that human behavior, disease awareness (through mass media) are important factors in the transmission of infectious diseases $[29,40]$ it would be more interessting, but challenging to incorporate these features in our contact tracing model for future works, as well as many other disease characteristics as follows:

- Development of a model in which the wearing of protective clothes and adequate equipments is the principal policy.

- Assessment the efficiency of both contact tracing and media coverage [65] to fight against EVD.

- Development of a similar model taking into account the delay inherent to contact tracing. Though, this will lead to delay differential systems, which can be 
more realistic but less mathematically tractable [60].

- Construction of a model for Ebola with contact tracing which takes into consideration the spillover event transmission from animal to human [17].

- Explicit incorporation of the vaccinated class of individuals as additional control measure [11].

- The use of multi-patch modeling approach to account for the circulation of EVD between villages, counties or countries as it was the case for the 2014 West Africa outbreak.

\section{Acknowledgments}

The first author (T.B.) acknowledges the financial support of the University of Pretoria Senior Postdoctoral Programme Grant (2017-2019). The authors will like to thank the two anonymous referees whose comments and suggestions helped to substantially improve the quality of this paper.

\section{References}

[1] F. B. Agusto, M. I. Teboh-Ewungkem, A. B. Gumel, Mathematical Assessment of the Effect of Traditionnal Beliefs and Customs on the Transmission Dynamics of the 2014 Ebola outbreaks, BMC 13 (96) (2015) 1-18.

[2] F. B. Agusto, Mathematical Model of Ebola Transmission Dynamics with Relapse and Reinfection, Math. Biosci. 283 (2017) 48-59.

[3] M. D. Ahmad, M. Usman, A. Khan, M. Imran, Optimal Control Analysis of Ebola Disease with Control Strategies of Quarantine and Vaccination, Infect Dis Poverty 5:72 (2016) 1-12.

[4] M. Ajelli, S. Parlamento, D. Bome, A. Kebbi, A. Atzori, C. Frasson, G. Putoto, D. Carraro, S. Merler, The 2014 Ebola Virus Disease Outbreak in Pujehun, Sierra Leone: Epidemiology and Impact of Interventions, BMC Medicine 13:281 (2015) 1-8.

[5] C. Althaus, Estimating the Reproduction Number of Ebola (EBOV) during outbreak in West Africa, PLOS Currents Outbreaks, doi: 10.1371/currents.outbreaks.91afb5e0f279e7f29e7056095255b288, (2014) 1-5.

[6] C. L. Althaus, N. Low, E. O. Musa, F. Schuaib, S. Gsteiger, Ebola Virus Disease Outbreak in Nigeria: Transmission Dynamics and Rapid Control, Epidemics 11 (2015) $80-84$.

[7] R. M. Anderson, R. M. May, Infectious Diseases of Humans: Dynamics and Control, Oxford, England: Oxford University Press (1991).

[8] R. M. Anderson, R. M. May, S. Gupta, Non-linear Phenomena in Host-Parasite Interactions, Parasitology, 99 Supp (1989).

[9] I. Area, H. Batarfi, J. Losada, J. J. Nieto, W. Shammakh, A. Torres, On a Fractional order Ebola Epidemic Model, Adv. Differ. Equ. 278 (2015) 1-12.

[10] I. Area, J. Losada, F. Ndaïrou, J. J. Nieto, D. D. Tcheutia, Mathematical Modeling of 2014 Ebola Outbreak, Math. Meth. Appl. Sci. 40 (2015) 6114-6122.

[11] I. Area, F. Ndaïrou, J. J. Nieto, C. J. Silva, D. F. M. Torres, Ebola Model and Optimal Controls with Vaccination Constraints, JIMO 278 (2017) 1-21.

[12] T. Attenborough, Modelling the Ebola Outbreak in West Africa and Community Responses, Complex University College London (2015), 1-21.

[13] K. Barley, Parameter Estimation and Mathematical Modeling of Visceral Leishmaniasis Transmission, Arizona State University, PhD thesis (2016) pp 56. 
[14] T. Berge, S. Bowong, J .S. -M. Lubuma, Global Staility of a two-patch Cholera Model with Fast and Slow Transmissions, Math. Comput. Sim. 133 (2017) 142-164.

[15] T. Berge, S. Bowong, J. M. -S.Lubuma, M. L. M. Manyombe, Modelling Ebola Virus Disease Transmissions with Reservoir in a Complex Virus Life Ecology, Math. Biosci. 15 (1) (2018) 21-56.

[16] T. Berge, M. Chapwanya, J. S. -M. Lubuma, Y. A. Terefe, A Mathematical Model

for Ebola Epidemic with Self-Protection Measures, J. Biol. Syst. 26(1) (2018) 107-132.

[17] T. Berge, J. S. -M. Lubuma, A. J. O. Tassé, H. M. Tenkam, Dynamics of HostReservoir Transmission of Ebola with Spillover Potential to Humans, EJQTDE (14) (2018) 1-32.

[18] Berman, Plemmons, Nonnegative Matrices in the Mathematical Sciences Academic, Press, New York (1979).

[19] G. Birkhoff, G-C. Rota, Ordinary Differential Equations, John Wiley \& Sons (1989).

[20] E. N. Bodine, C. Cook, M. Shorten, The Potential impact of a Prophylactic Vaccine for Ebola virus disease in Sierra Leone, Math. Biosci 15 (2018) 337-359.

[21] C. S. Bornaa, O. D. Makinde, I. Y. Seini, Eco-Epidemiological Model and Optimal Control of Disease Transmission Between Humans and Animals, Commun. Math. Biol. Neurosci. 2015:26 (2015) 2052-2541.

[22] A. Brettin, R. Rossi-Goldthorpe, K. Weishaar, I. V. Erovenko, Ebola could be Eradicated through Voluntary Vaccination, R. Soc. Open Sci 5:171591 (2017) 1-12.

[23] C. Browne, X. Huo, P. Magal, M. Seydi, O. Seydi, G. Webb, A Model of the 2014 Ebola Epidemic in West Africa with Contact Tracing, Plos Currents Outreaks, doi: 10.1371/currents.outreaks.846b2a31ef37018b7d1126a9c8adf22a (2015) 1-20.

[24] D. Butler, How Disease Detectives are fighting Ebola's Spread, nature, www.nature.com/news/how-disease-detectives-are-fighting-ebola-s-spread-1.16069, 02 October 2014.

[25] G. Caleo, J. Duncombe, F. Jephcott, K. Lokuge, C. Mills, E. Looijen, F. Theohakary, R. Kremer, K. Kleijer, J. Squire, M. Lamin, Beverley Stringer, H. A. Weiss, D. Culli, G. L. D. Tanna, J. Greig, The Factors Affecting Household Transmission Dynamics and Community Compliance with Ebola Control Measures: a Mixed-Methods Study in a Rural Village in Sierra Leone, BMC Public Health 18:248 (2018) 1-13.

[26] A. Camacho, A. J. Kucharski, S. Funk, J. Breman, P. Piot, W. J. Edmunds, Potential for Large Outbreaks of Ebola Virus Disease, Epidemics 9 (2014) 70-78.

[27] Cdc.gov, Transmission-Ebola Hemorrhagic Fever, CDC (2015), http://www.cdc.gov/vhf/ebola/transmission/index.html.

[28] G. Chowell, N. W. Hentgartner, C. Castillo-Chavez, P. W. Fenimore, J. M. Hyman, The Basic Reproductive Number of Ebola and the Effects of Public Health measures: The cases of Congo and Uganda, J. Theor. Biol. 2004 (229) 119-126.

[29] J. R. Conrad, L. Xue, J. Dewar, J. M. Hyman, Modeling the impact of Behavior Change on the Spread of Ebola, Mathematical and Statistical Modeling for Emerging and Re-emerging Infectious Diseases, doi: 10.1007/978-3-319-40413-4_2, (2016).

[30] Contact Tracing during an outbreak of Ebola Virus Disease, World Health Organization Regional Office for Africa, who.int./csr/resources/publications/ebola/contacttracing-during-outbreak-of ebola.pdf, (2014) 1-19.

[31] K. Dammers, Quarantine and Isolation Law: The Basics, Public Health Law Program U.S Centers for Disease Control and Prevention 55-77.

[32] A. Delamou, T. Delvaux, A. M. El Ayadi, A. H. Beavogui, J. Okumura, W. V. Damme, V. De Brouwere, Public Health Impact of the 2014-2015 Ebola Outbreak in West Africa: Seizing Opportunities for the Future, BMJ Global Health 2:e000202 (2017) 1-3. 
[33] O. Diekmann, J. A. P. Heesterbeck, J. A. J. Metz, On the Definition and the Computation of the Basic Reproduction Ratio $\mathcal{R}_{0}$ in Models for Infectious Diseases in Heteregeneous Populations, J. Math. Biol. 28 (1990) 365-382.

[34] M. O. Durojaye, I. J. Ajie, Mathematical Model of the Spread and Control of Ebola Virus Disease, J. Appl. Math. Comput. 7 (2017) 23-31.

[35] Ebola "threat to world security"-UN Security Council, $B B C$, www.bbc.com/news/world-africa-29262968, 19 September 2014.

[36] L-Q. Fan, Y. Yang, W-C. Cao, Transmission Dynamics of Ebola Virus Disease and Intervention Effectiveness in Sierra Leone, PNAS 113 (2016) 4488-4493.

[37] F. O. Fasina, A. Shittu, D. Lazarus, O. Tomori, L. Simonsen, C. Viboud, G. Chowell, Transmission Dynamics and Control of Ebola Virus Disease Outbreak in Nigeria, Euro. Surveil. 19 (40) (2014) 1-8.

[38] D. Fisman, E. Khoo, A. Tuite, Early Epidemic Dyamics of the Western African 2014 Ebola outbreak: Estimates Derived with a Simple two Parameter Model, PLOS Currents Outbreaks, doi: 10.1371/currents.outbreaks.89c0d3783f36958d96ebbae97348d571 (2014) 1-19.

[39] D. Gao, Transmission Dynamics of some Epidemiological Patch Model, University of Miami, (2012), phd thesis.

[40] L. Garrett, Ebola: Story of an Outbreak, Hachette Books, New York (2014).

[41] B. Gholipour, American Ebola Survivors are Likely Immune to Virus Strain Now, Live Science, https://www.livescience.com/47511-are-ebola-survivors-immune-html (2014).

[42] A. Gideon, I. Miranda, Teboh-Ewungkem, A Mathematical Model with Quarantine states for the dynamics of Ebola Virus Disease in Human Populations, Comput. Math. Methods Med. 2016 (2016) 1-29.

[43] A. B. Gumel, S. Ruan, T. Day, J. Watmough, F. Brauer, P. van den Driessche, D.Gabrielson, C. Bowman, M. E. Alexander, S. Ardal, J. Wu, B. M. Sahai, Modelling Strategies for Controlling SARS Outbreaks, The Royal Society 271 (2004) 2223-2232.

[44] H. Gumsova, A. Shittu, M. Sanbul, H. Leblebicioglu, Ebola Virus Disease and the Veterinary Perspective, BMC 14 (30) (2015) 1-5.

[45] R. Hamilton, Ebola Crisis: The Economic Impact, www.bbc.com/news/business28865434.

[46] H. W. Hetcote, The Mathematics of Infectious Diseases, SIAM 2000 (42) 599-653.

[47] M. Imran, A. Khan, A. R. Ansari, Modeling Transmission Dynamics of Ebola Virus Disease, IJB 10 (2017) 1750057-1-35.

[48] Issues to Consider Isolation \& Quarantine, NACCHO https://ftp.cdc.gov/pub/phlpprep/Legal\%20Preparedness\%20for\%20Pandemic\%20 Flu/8.0\%20-\%20Non-Governmental\%20Materials/8.5\%20NAACHO\%20I\&O.pdf, (2006) 1-8.

[49] B. Ivorra, D. Ngom, A. M. Ramos, A Mathematical Model to Predict the Risk of Human Disease Spread between Countries-Validation and Application to 2014-2015 Ebola Virus Disease Epidemic, Bull. Math. Biol. 77 (2015) 1668-1704.

[50] D. Kalajdzievska, M. Y. Li, Modelling the Effects of Carriers on the Transmission Dynamics of Infectious Diseases, Math. Biosc. 8 (2011) 711-722.

[51] W. O. Kermack, A. G. Mckendrick, Contributions to the Mathematical Theory of Epidemics, Proc. R. SOC. Lond. A 115 (1927).

[52] M. A. Kimaro, E. S. Massawe, D. O. Makinde, Modelling the Optimal Control of Transmission Dynamics of Mycobacterium Ulceran Infection, OJEpi 5(2015) 229-243.

[53] A. J. Kucharski, R. M. Eggo, C. H. Watson, A. Camacho, S. Funk, J. Edmungs, Effectiveness of Ring Vaccination as Control Strategy for Ebola Virus Disease, Emerg. Infect. Dis. 22 (2016) 105-108. 
[54] J. P. LaSalle, The Stability of Dynamical Systems, SIAM, (1976).

[55] V. P. Latha, F. A. Rihan, R. Rakkiyappan, A Fractional-Order Delay Differential Model for Ebola Infection and CD8 ${ }^{+}$T-Cells Response: Stability Analysis and Hopf Bifurcation, IJB 10 (2017) 1750111-1-22.

[56] J. Legrand, R. F. Grais, P. Y. Boelle, A. J. Valleron, A. Flahault, Understanding the Dynamics of Ebola Epidemics, Epidemiol. Infect. 135 (2007) 610-621.

[57] X. Liao, L. Wang, P. Yu, Stability of Dynamical Systems, Monograph Series on Nonlinear Science and Complexity 5 (2007).

[58] S. Marino, I.B. Hogue, C.J. Ray and D.E. Kirschner, A Methodology for Performing Global Uncertainty and Sensitivity Analysis in Systems Biology, J. Theor. Biol. 254 (2008) 178-196.

[59] S. Merler, M. Ajelli, L. Fumanelli, S. Parlamento, A. P. Y. Piontti, N. E. Dean, G. Putoto, D. Carraro, I. M. Longini, J. M. E. Halloran, A. Vespignani, Containing Ebola at the Source with Ring Vaccination, PLOS Negl. Trop. Dis 10 (11) (2016) 1-11.

[60] J. Müller, B. Koopmann, The Effect of Delay on Contact Tracing, Math. Biosci. 282 (2016) 204-214.

[61] R. Narra, J. Sobel, C. Piper, D. Gould, N. Bhadelia, M. Dott, A. Flore, W. A. Fisher, M. J. Frawley, P. M. Griffin, D. Hamilton, B. Mahon, S. K. Pillai, E. F. Veltus, R. Tauxe, M. Jhung, CDC Safety Training Course for Ebola Virus Disease Healthcare Workers, Emerg. Infect. Dis. 23(2017) S217-S224.

[62] D. Ndanguza, J. M. Tchuenche, H. Haario, Statistical data analysis of the 1995 Ebola outbreak in the Democratic Republic of Congo, Afr. Mat. 24 (2013) 55-68.

[63] V. K. Nguyen, R. Mikolajczyk, E. A. Hernandez-Vargas, High-Resolution Epidemic Simulation Using Within-Host Infection and Contact Data, Research, doi: 10.1101/133421 (2018) 1-22.

[64] R. Nistal Riobello, On some new Mathematical Models for Infective Diseases: Analysis, Equilibrium, Positivity and Vaccination Controls, University of Basque Country Leoia, (2015), PhD thesis, 148 pages.

[65] S. D. D. Njankou, Modelling the Potential Role of Control Strategies of Ebola Virus Disease Dynamics, University of Stellenbosch, South Africa, (2015), phd thesis, P.60.

[66] A. Pandey, K. E. Atkins, J. Medlock, N. Wenzel, J. P. Townsend, J. E. Childs, T. G. Nyenswah, M. L. Ndeffo-Mbah, A. P. Galvani, Strategies for Containing Ebola in West Africa, NIH 346 (6212) (2014) 991-995.

[67] S. Petti, C. Protano, G. A. Messano, C. Scully, Ebola Virus Infection Among Western Healthcare Workers Unable to Recall the Transmission Route, BioMed Research International, 2016 (2016) 1-5.

[68] Phenyo, E. Lekone, B. F. Finkendstädt, Statistical Inference in a Stochastic Epidemic SEIR Model with Control Intervention: Ebola as a case of Study, Biometrics 62 (4) (2006) 1170-1177.

[69] A. Rachah, D. F. M. Torres, Optimal Control Strategies for the Spread of Ebola in West Africa, J. Math. Anal. 7 (2016) 102-114.

[70] S. M. A. Rahman, Study of Infectious Diseases by Mathematical Models: Predictions and Controls, Western University, phd thesis (2016).

[71] A. Rădulescu, J. Herron, Ebola Impact and Quarantine in a Network Model, AJUR 13 (2016) 21-35.

[72] C. M. Rivers, E. T. Lofgren, M. Marathe, S. Eubank, B. L. Lewis, Modeling the Impact of Interventions on an Epidemic of Ebola in Sierra Leone and Liberia, PLOS currents Outbreaks, doi: 10.1371/ currents.outbreaks.fd38dd85078565450b0be3fcd78f5ccf (2014) 1-9.

[73] D. Salem, R. Smith, A Mathematical Model of Ebola Virus Disease: Using Sensitivity 
Analysis to Determine Effective Intervention Targets, SCS (2016) 16-23.

[74] F. Shuaib, R. Gunnala, E. O. Musa, F. J. Mahoney, O. Oguntimehin, P. M. Nguku, S. B. Nyanti, N. Knight, N. S. Gwarzo, O. Idigbe, A. Nasidi, J. F. Vertefeuille, Ebola Virus Disease Outbreak, CDC 63(39) (2014) 867-872.

[75] T. Sug Do, Y. S. Lee, Modeling the Spread of Ebola, Osong Public Health Res Perpect $7(2016)$ 43-48.

[76] H. R. Thieme, Persistence under Relaxed Point-Dissipativity (with Applications to an Endemic Model), J. Math. Anal. 24 (1993) 407-435.

[77] S. Towers, O. Patterson-Lomba, C. Castillo-Chavez, Temporal Variations in the Effective Reproduction Number of the 2014 West Afica Ebola outbreak, PLOS Currents Outbreaks, doi:10.1371/currents.outbreaks.9e4c4294ec8ce1adad283172b16bc908 (2014) 1-13.

[78] B. Tsanou, J. M.-S. Lubuma, G. M. Moremedi, N. Morris, R. Kondera-Shava, A Simple Mathematical Model for Ebola in Africa, J. Biol. Dyn. 11:1 (2016) 42-74.

[79] B. Tsanou, S. Bowong, J. Mbang, J. Lubuma, Assessing the Impact of the Environmental Contamination on the Transmission of Ebola Virus Disease (EVD), J. Appl. Math. Comput. 55 (2017) 205-243.

[80] T. W. Tulu, B. Tian, Z. Wu, Modeling the Effect of Quarantine and Vaccination on Ebola Disease, Adv. Differ. Equ 2017:178 (2017) 1-14.

[81] P. Van den Driessche, J. Watmough, Reproduction Numbers and Sub-threshold Endemic Equilibria for Compartment Models of Disease Transmission, Math. Biosci. 180 (2002) 29-48.

[82] X. -S. Wang and L. Zhong, Ebola Outbreak in West Afica : Real-time Estimation and Multiple-Wave Prediction, Math. Biosci. 12 (5) (2015) 1055-1063.

[83] S. Wiafe, E. Veilleux-Gravel, R. Smith, Using Sensitivity Analysis to Examine the Effects of an Ebola Vaccine, SCS 12 (2017) 61-72.

[84] World Bank Group, The Economic Impact of the 2014 Ebola Epidemic: Short and Medium Term Estimates for West Africa, https://openknowledge.worldbank.org/en/region/afr/publication/theeconomic-impact-ofthe-2014-ebola-epidemic-short-and-medium-term-estimates-for-west-africa, 8-10-2014, $1-109$.

[85] World Health Organization, Health Worker Ebola Infections in Guinea, Liberia and Sierra Leone, WHO/EVD/SDS/REPORT/2015.1 (2015), 1-16.

[86] I. York, Can a Person who has Contracted the Ebola Virus Catch Ebola again if they Survived the Infection?, Quora, www.quora.com/can-a-person-who-has-contractedthe-Ebola-virus-catch-Ebola-again-if-they-survived-the-first-infection (2014).

[87] J. Zhang, Infectious Disease Model with the Latent Period and Quarantine and Comparison of Drug Distribution Scheme based on Ebola Virus, ISSS (2015) 362-366.

[88] J-M. Zhu, L. Wang, J-B. Liu, Eradication of Ebola Based on Dynamic Programming, Comput. Math. Methods Med. 2016 (2016) 1-10. 